\title{
A CLASS OF HARMONIC FUNCTIONS IN THREE VARIABLES AND THEIR PROPERTIES
}

\author{
BY \\ STEFAN BERGMAN
}

\section{INTRODUCTION}

1. General remarks on operators generating solutions of partial differential equations in two variables and harmonic functions in three variables. In order to study the properties of real solutions of partial differential equations

$$
\mathbf{L}(U) \equiv \frac{\partial^{2} U}{\partial x_{1}^{2}}+\frac{\partial^{2} U}{\partial x_{2}^{2}}+A \frac{\partial U}{\partial x_{1}}+B \frac{\partial U}{\partial x_{2}}+D U=0,
$$

integral operators of a certain type have recently been applied in papers [3, $4]\left({ }^{1}\right)$, and other publications indicated in [4].

By means of these operators it was shown that a duality exists between certain classes $\mathcal{C}(\mathbf{E})$ of complex solutions, $u$, of an equation $\mathrm{L}$ and analytic functions $f(z)$ of a complex variable $z=x_{1}+i x_{2}$.

These complex solutions were obtained in the following manner:

To every differential equation $\mathrm{L}$ whose coefficients $A, B, D$ are entire functions of $x_{1}$ and $x_{2}$, integral operators $\left({ }^{2}\right)$

$$
\begin{aligned}
u\left(x_{1}, x_{2}\right)=\mathrm{P}(f) & \equiv \int_{-1}^{1} \mathrm{E}(z, \bar{z}, t) f\left[z\left(1-t^{2}\right) / 2\right] d t /\left(1-t^{2}\right)^{1 / 2}, \\
z & =x_{1}+i x_{2}, \quad \bar{z}=x_{1}-i x_{2},
\end{aligned}
$$

have been determined which transform analytic functions, $f$, into complex solutions, $u$, of $\mathrm{L}$ such that when $f$ ranges. over the totality of analytic functions, $\operatorname{Re}[\mathrm{P}(f)]$ ranges over the totality of real solutions of $\mathrm{L}(\mathrm{Re}=$ the real part).

We have a certain amount of freedom in choosing $\mathbf{E}$ for a given equation $\mathbf{L}$,

Presented to the Society, October 28, 1944, under the title Representation of potentials of electric charge distributions; received by the editors June 30, 1945.

(1) The numbers in brackets refer to the bibliography. The present paper does not presuppose knowledge of previous publications of the author.

(2) The relation $u=\mathrm{P}(f)$ can be interpreted as a mapping (in the function space) of the class of analytic functions, $f$, into the class, $\mathcal{C}(\mathrm{E})$, of complex solutions $u$ of $\boldsymbol{L}$. It should be stressed that in this approach the domain in the $x_{1} x_{2}$-plane in which the functions $u\left(x_{1}, x_{2}\right)$ are considered is not a fixed region. The representation (1.2) in many instances is valid in any simply connected domain which includes the origin and in which the function $u$ under consideration is regular. (It may well happen that this domain is the whole plane or a Riemann surface over the whole plane.) A further feature of this approach is that the use of operators yields results concerning the behaviour of $u$ outside the domain, in which it is represented by (1.2). 
that is, using integral operators (1.2) and conveniently changing the function $\mathbf{E}$, we can obtain various classes, $\mathcal{C}(\mathbf{E})$, of complex solutions of the same equation $\left({ }^{3}\right) \mathbf{L}$. By a convenient choice of $\left({ }^{4}\right) \mathbf{E}$ we obtain a certain class $\mathcal{C}(\mathbf{E})$ of complex solutions $u$, where each $u$ is connected in a comparatively simple manner with the corresponding function $f$. See [4] and the papers indicated there.

Since the theory of analytic functions of a complex variable has been extensively studied, the use of the integral operator described above enables us to "translate" many results of this theory to the case of above-mentioned complex solutions of $\mathbf{L}$, and consequently use them for the study of real solutions of $\mathbf{L}$. The class of functions $u$ mentioned above is particularly suitable for transferring certain essential properties of analytic functions to the theory of partial differential equations. On the other hand for the derivation of some special properties of real solutions it is more convenient to use other classes of complex solutions than the class mentioned above. Further, using generating functions different from those of the first kind we obtain various new theorems concerning the $U$ 's. These results of ten are different from those which have been obtained by analogous considerations but using the generating function of the first kind. These are some of the reasons why the derivation and investigation of all possible operators which transform analytic functions of a complex variable into solutions of $\mathbf{L}$ is of considerable interest.

The next step in the development of this theory is the application of a similar method to equations in three variables. The Laplace equation in three variables represents the simplest case in this study.

Investigating solutions of this equation we can consider either a single harmonic function $h\left(x_{1}, x_{2}, x_{3}\right)$ or a "harmonic vector" $H$ (that is, a triple of functions), whose components satisfy the relations

$$
\nabla \cdot \mathrm{H}=0, \quad \nabla \times \mathrm{H}=0 .
$$

We note that in the case of two variables the relations (1.3) are the CauchyRiemann equations.

There exists a simple relation between the theory of harmonic vectors and that of harmonic functions, since by applying the operator $\nabla$ one can obtain a harmonic vector from a single harmonic function.

Another alternative consists in considering either real or complex harmonic functions and vectors. In physical applications we need, of course, real functions; but in the process of investigation it is sometimes more convenient to operate with complex functions.

(3) To each choice of $\mathbf{E}$ corresponds a certain relation between the real and imaginary parts of $u, U$ and $V$, respectively: If therefore $u_{1}$ and $u_{2}$ are two complex solutions of $\mathrm{L}$, which have the same real part but which belong to two different classes, then, in general, the imaginary parts, $V_{1}$ and $V_{2}$, of $u_{1}$ and $u_{2}$, respectively, will be different.

(4) This function $\mathbf{E}$ is of ten denoted as the generating function of the first kind. 
As has been indicated before in the study of different properties of real solutions of an equation it may be more convenient to use one operator rather than another.

This is particularly true in considering equations in three variables. In $[1,2]$ the author has introduced the operator $\left(^{5}\right)$

$$
\begin{gathered}
h\left(x_{1}, x_{2}, x_{3}\right)=\mathrm{P}_{2}(f) \equiv \int_{8} f(Z, \zeta) d \zeta, \\
Z=x_{1}+i\left(\zeta+\zeta^{-1}\right) x_{2} / 2+\left(\zeta-\zeta^{-1}\right) x_{3} / 2
\end{gathered}
$$

which generates harmonic functions from analytic functions of two complex variables $Z$ and $\zeta$. Here $R$ is a closed curve in the complex $\zeta$ plane. As we shall explain in more detail in the next section, it is of interest in the three-dimensional case to investigate in addition to (1.4) other types of integral operators which generate harmonic functions.

In the present paper the operator $\left.{ }^{6}\right)$

$$
h\left(x_{1}, x_{2}, x_{3}\right)=\mathrm{H}(\mathrm{E})=\int_{\mathfrak{R}} \mathrm{E}\left(x_{1}, x_{2}, x_{3} ; \zeta\right) f(\zeta) d \zeta
$$

which generates harmonic functions and which is different from (1.4) is considered. Here

$$
\mathrm{E}\left(x_{1}, x_{2}, x_{3} ; \zeta\right)=\left\{\sum_{k=1}^{3}\left[x_{k}-u_{k}(\zeta)\right]^{2}\right\}^{-1 / 2}
$$

$u_{k}(\zeta), k=1,2,3$, being some fixed rational functions of $\zeta$.

2. The theory of operators in the case of harmonic functions in three variables and the theory of integrals of algebraic functions. In an approach based on the introduction of integral operators the following two questions present themselves almost immediately:

(1) The determination of the domain $\left({ }^{7}\right), \mathfrak{a}$, in which the operator, that is, the integrals (1.2), (1.4), (1.5), respectively, in the cases under consideration, represents a solution of the equation.

(2) The determination of means ior analytic continuation of the solution outside the domain $\mathfrak{a}$ (or a connected subdomain of $\mathfrak{a}$ in which a solution is

(5) (1.4) is a generalization of the Whittaker operator, which we obtain by substituting $\zeta=e^{i t}$ in (1.4), and specializing $\&$ to be the unit circle. It should be stressed that in the approach developed here and in previous papers of the author the interest lies not in the operators themselves but mainly in their use as a tool for translating results from one theory to the other and, in the ultimate goal, as a means for studying real solutions of $\mathrm{L}$.

(b) The function $f$ is denoted as the associate function of $h$ with respect to the operator $\mathbf{E}$.

(7) Clearly it can happen that the domain in which the integral operator exists consists of several disconnected regions, in each of which the integral operator represents another function, or another function element of the same function. 
defined by the integral operator) into the whole domain of regularity of the solution under consideration.

A closer examination of operators (1.4) and (1.5) leads in problem (2) to the conclusion that in the case of harmonic functions it is useful to consider not all harmonic functions generated by the operator when the function $f$ appearing in the integrand ranges over the entire class of analytic functions of a complex variable, but rather certain subclasses of harmonic functions, namely those for which the results of the theory of analytic functions of a complex variable possess a particularly simple "translation" with respect to the operator to be employed.

In order to clarify what is meant by this last statement it will be useful to compare (from a certain point of view) the similarities and differences that occur in the transition, by operators, from analytic functions to solutions of differential equations of elliptic type in two variables with those that occur in the passage to harmonic functions in three variables.

In passing from analytic functions of one complex variable to harmonic functions in three real variables, we increase the number of variables, rendering the corresponding "translation" of the results of analytic function theory more difficult of interpretation, and, as a consequence, these "translated" statements often assume a form which is quite different from that in the case of harmonic functions of two variables.

On the other hand in the case of equations $\mathbf{L}$, see (1.1), in (1.2) a generating function $\mathrm{E}(z, \bar{z}, t), \bar{z}=x_{1}-i x_{2}$, appears which has a transcendental character and which considerably changes the behaviour of the obtained functions at infinity; in the case of harmonic functions in three variables there exist operators whose generating function is either 1 , see (1.4), or an algebraic function of $x_{1}, x_{2}, x_{3}$, see (1.5).

This fact suggests considering at first the subclass of harmonic functions whose associates, $f$, are rational or algebraic functions. The study of the subclass obtained by (1.4) or (1.5) becomes in this case the investigation of integrals of algebraic functions, which in addition to the (complex) integration variable $\zeta$ depend upon three real parameters, namely the cartesian coordinates $x_{1}, x_{2}, x_{3}$ of the space. The classical theory of integrals of algebraic functions is a highly developed branch of analysis, and the investigation of the subclass of harmonic functions obtained in the manner described above reduces itself to interpretation of classical results on integrals of algebraic functions.

As a consequence of this fact, the study of the various operators that may be employed in the case of harmonic functions in three variables forms a particularly interesting study, since in this case there correspond to the totality of algebraic functions of one variable many different classes of harmonic functions in three variables. For instance the operator (1.5) (when $f$ ranges over the totality of algebraic functions) yields a harmonic function with singulari- 
ties along segments $\left({ }^{8}\right)$ of rational curves $\left({ }^{9}\right)$, while the operator (1.4) yields in the same case only functions which are, as a rule, singular along closed curves.

3. A general survey of methods and results. This paper is an investigation of certain properties of harmonic functions $h\left(x_{1}, x_{2}, x_{3}\right)$ of three real variables generated from the class of analytic functions $f(\zeta)$ of one complex variable by means of the integral formula

$$
h(\mathrm{X})=\int_{\mathbf{\Omega}} \mathrm{E}(\mathrm{X} ; \zeta) f(\zeta) d \zeta
$$

see (1.5). In this formula $\mathrm{X}$ represents the number-triple $\left(x_{1}, x_{2}, x_{3}\right)$ and may be interpreted as a position vector in the space $\mathfrak{r}$ of three dimensions; $\zeta$ is a complex parameter ranging over the complex $\zeta$-plane; $R$ is a specified curve in the $\zeta$-plane; $\mathrm{E}$ is given by (1.6), and $f(\zeta)$ is an analytic function of $\zeta$. It will be assumed hereafter that $\zeta$ is restricted to the regularity domain of $f$. It is evident that $\mathbf{E}$ is a complex harmonic function, so that the integral $h(\mathrm{X})$ is a complex harmonic function in three variables.

In addition to problems (1) and (2) of $\$ 1$, this paper considers the question of determining the special properties of $h$ which result from the particular form of $f$-namely that $f$ is a rational function (see the remarks in $\S 2$ ).

The integrand of (1.7) is an analytic function of $\zeta$ which depends on the three real parameters $\left(x_{1}, x_{2}, x_{3}\right)$, that is, it represents a three-parameter family of complex analytic functions. A function of this family belongs to each point $X \in \mathfrak{r}$, the euclidean three-dimensional space. For a fixed $X$, the integrand is a two-valued analytic function of $\zeta$, defined on a Riemann-surface of two sheets, denoted by $\Re(\mathrm{X})$. The number of branch points depends on the form of the $u_{i}(\zeta)$, as well as on the particular point X. As $\mathrm{X}$ ranges over $\mathfrak{r}$, $\Re(\mathrm{X})$ changes continuously, that is, the branch points of $\Re(\mathrm{X})$ continuously change their positions in the $\zeta$-plane. It may happen that two distinct branch points coincide for certain values of $X$. Denote the set of all points $X$ for which two branch points coincide by 8 ; the set 8 is henceforth to be excluded from consideration. If there are any values of $\mathrm{X}$ for which a factor of $\mathrm{E}$ vanishes identically, independently of $\zeta$, such points are also to be included in 8 and therefore excluded from the domain of definition of (1.7) ( $\$ 1$ of chap. II). Furthermore, the integrand becomes infinite at all the zeros of the quantity under the square root (1.6). The set of points $\mathrm{X}$ for which this happens lies on a surface $\Im$, and clearly the integral (1.7) is not defined on $\Im$. Consequently the integral representation (1.7) of $h$ is defined in the domain $\mathfrak{a}=\mathfrak{r}-\mathfrak{b}-\mathfrak{\Im}$. The form and mutual relations of the singular lines 8 and the

(8) The harmonic functions which are singular along a segment of a rational curve in the $x_{1}, x_{2}, x_{3}$ space represent a very important subclass of harmonic functions. They admit the physical interpretation as potentials of linear charge distribution along segments mentioned above.

( ${ }^{\ominus}$ A curve is said to be a rational curve if it can be represented in the form $x_{k}=u_{k}(t)$, where $u_{k}$ are appropriate real rational functions of the real variable $t$. 
discontinuity surface $\Im$ can be explicitly determined when the functional forms of $u_{i}(\zeta)$ and of $f(\zeta)$ are given ( $\$ 3$ of chap. II). The first problem may now be considered as solved in principle $\left({ }^{10}\right)$.

In order to continue $h(\mathrm{X})$ outside $\mathfrak{a}$ it is necessary to find out how the surface $\Im$ is determined by the path of integration, $\mathbb{R}$. In $\$ 4$ of chap. II, it is shown how to construct $\Im$ when $\mathfrak{R}$ is given. As $\mathfrak{R}$ varies $\Im$ varies also (changing the domain $\mathfrak{a}$ ). The boundary curve of $\Im$ does not vary however, since the end points of $\mathfrak{R}$ are kept fixed.

For a fixed point $\mathrm{X}$ the value of the integral is the same no matter how $\mathbb{R}$ is deformed, provided that it passes over no singularity, because the integrand is an analytic function of $\zeta$. By varying $\mathfrak{R}$ we vary $\Im$, and therefore $\mathfrak{a}$. This process defines the harmonic function $h(\mathrm{X})$ at points which were not included in the initial domain of regularity of the integral representation; therefore this is a method of analytic continuation $\left({ }^{11}\right)$. In general, a point of $\Im$ (not on its boundary) is a regularity point of $h(\mathrm{X})$ ( $\$ 5$ of chap. II). On the other hand, a surface $\Im$ may contain points over which $h(\mathrm{X})$ cannot be continued. These singularities are (in general) independent of the particular integral representation and may be regarded as singularities of the function $h$ which can not be removed by analytic continuation.

Remark 1.1. It is clear that in the general case $h(\mathrm{X})$ defined by analytic continuation is a many-valued function of $\mathrm{X}$. If $h(\mathrm{X})$ is continued analytically around one of the singular curves, it may happen that $h$ will not return to the same value from which we started. This is analogous to the situation in the theory of many-valued analytic functions, and an analogous device may be used in treating it. A generalization of the Riemann surface may be defined, each "sheet" consisting of a region in space of three dimensions, called a space-sheet. Different sheets which may be finite or infinite in number and extent are joined at the singularity curves, which here play the part of "branch-lines."

Chapter III is devoted to the special case where $f(\zeta)$ is a rational function. The integral formula (1.7) for $h(\mathrm{X})$ then becomes a hyperelliptic integral in $\zeta$ depending on the parameter $x_{1}, x_{2}, x_{3}$. The methods of Weierstrass for the theory of algebraic functions are employed because they lead to explicit formulas from which it can be seen how the functions defined by the integral depend on $\mathrm{X}$ ( $\$ 1$ of chap. III). The Riemann surface $\Re(\mathrm{X})$ which is determined by $\mathbf{E}(X ; \zeta)$ possesses an even number, say $2 \rho+2$, of branch points; its genus is $\rho$. (Clearly $\rho$ is independent of X.) By a linear transformation (3.1) one of

(10) The reader is cautioned to distinguish carefully between the harmonic function $h(\mathrm{X})$ and its particular representation in the form of the integral (1.7). In general, the integral represents $h(\mathrm{X})$ only in a part of the full domain of regularity (which is found by analytic continuation). See below.

(11) In $\$ 6$ of chap. II we use this device to obtain a formula for the saltus at a point of the discontinuity surface $\Im$. 
the branch points is removed to infinity. Call the modified Riemann surface $\mathfrak{W}(\mathrm{X})$ ( $\$ 2$ of chap. III). Now cut $\mathfrak{W}(\mathrm{X})$ by $\rho$ loop-cuts and $\rho$ crosscuts in the manner described in $\$ 3$ of chap. III and introduce the periods of the normal integrals of the first and the second kind of the surface $\mathfrak{B}(\mathrm{X})$. These periods [period functions $\omega_{\alpha \beta}(\mathrm{X}), \eta_{\alpha \beta}(\mathrm{X})$ ] are infinitly many-valued functions of $\mathrm{X}$. They become single-valued functions if we consider them in a suitably constructed domain of the type described in Remark 1.1 ( $\$ 4$ of chap. III). Now we form the Weierstrass $H$-functions ( $\$ 5$ of chap. III), with the aid of which the normal integrals of the first, second and third kind may be defined. These integrals depend on the end points of the integration path $\mathbb{R}$ as well as on $\mathrm{X}$. Then in $\S 6$ of chap. III, $h(\mathrm{X})$ is expressed as a linear combination of normal integrals with coefficients which are algebraic functions of X. Finally in $\$ 7$ the theta-functions $\theta\left(u_{1}, \cdots, u_{p} ; X\right)$ are introduced, whose parameters (not appearing, as arguments of $\theta$ ) are period functions of $\$ 4$ of chap. III. $\rho$ is the genus of $\mathfrak{W}(\mathrm{X})$.

It is one of the most important achievements of the theory of algebraic functions that the normal integrals of the second and third kind can be expressed explicitly in a closed form in terms of integrals of first kind, the $\theta$-functions, and the first derivatives of $\theta$-functions. As a consequence of this theorem we obtain in $\$ 7$ the following result: Consider the subclass of $\delta\left(E, \zeta_{0}, \zeta_{1}\right)$ of harmonic functions which one obtains fixing $\mathrm{E}$, see (1.6), and the end points $\zeta_{0}$ and $\zeta_{1}$, but permitting $f$ to range over the totality of rational functions in $\zeta$. Every subclass $\delta\left(E, \zeta_{0}, \zeta_{1}\right)$ possesses a finite basis, more precisely, there exists a finite number of (transcendental) functions in $\mathrm{X}$ which can be determined from $\mathrm{E}, \zeta_{0}$ and $\zeta_{1}$ and such that every function $h(\mathrm{X})$ of the subclass can be represented as a (closed) algebro-logarithmic expression involving the above $\theta$-functions, their derivatives with respect to $u_{\alpha}$, and some algebraic functions in $\mathrm{X}$ as well as finitely many functions mentioned above.

4. Concluding remarks. In addition to investigating a class of harmonic functions which play an important role in physical application, the purpose of the present paper consists in developing an approach to three-dimensional harmonic functions in the framework of the theory of operators.

As we have emphasized there exist operators similar to (1.4) and (1.5) which transform analytic functions of a complex variable into classes of solutions of partial differential equations of elliptic type in three variables. (They degenerate in the case of the Laplace equation to (1.4) and (1.5), respectively.) It is of interest to use these operators to study not only the "translation rule" which governs the transition from analytic functions of one complex variable to the class of (complex) solutions of these equations, but also the relations which exist between the latter class and classes of harmonic functions in three variables.

The present paper and $[1,2]$ form one of the bases for investigations of this kind. 
The considerations of chapter III assume that the associate $f(\zeta)$ is a rational function of $\zeta$.

In order to generalize them to the case where the associate $f(\zeta)$ is an entire or meromorphic function of $\zeta$ it would be at first necessary to develop a theory of integrals of entire or meromorphic functions of a complex variable $\left({ }^{12}\right)$.

The present knowledge of the theory of analytic functions of a complex variable makes the development of such a theory quite feasible.

In this connection I should like to indicate one further interesting possibility of applying the method employed in the present paper.

Suppose that $f(\zeta)$ is an entire function, that we fix the initial point, say $\zeta_{0}$ of the integration curve, and that we consider the behaviour of our functions as a function of the end point, $\zeta$, of $\mathbb{R}$ when $\zeta \rightarrow \infty$. If the function $\mathbf{E}$ and the series development of $h\left(x_{1}, x_{2}, x_{3}\right)$ at the origin are given, then it is possible from these data to determine the coefficients of the associate function $f(\zeta)$. Since we assume that $f(\zeta)$ is an entire function, the classical theorems give us the upper bound for the growth of $f(\zeta)$, and consequently upper bound of the integral (1.5) at a fixed point $x_{1}, x_{2}, x_{3}$, where the end point of the integration curve goes to infinity.

Harmonic functions generated in a way similar to that developed in the present paper were considered in the literature. (We wish especially to indicate the important paper by Sommerfeld [6] where functions of the form

$$
\int_{\alpha=0}^{2 \pi} \frac{1}{\left[\left(x_{1}-\rho \cos \alpha\right)^{2}+\left(x_{2}-\rho \sin \alpha\right)^{2}+\left(x_{3}-z\right)^{2}\right]^{1 / 2}} \frac{e^{i \phi^{\prime} / n}}{e^{i \alpha / n}-e^{i \phi^{\prime} / n}} d \alpha
$$

are considered.)

However the papers mentioned above are devoted to quite different questions from those studied here, and have almost nothing in common with our considerations.

II. The PRoperties of The INTEgRaL (1.5)

1. Notation. An exact description of the operator. In the following, let

$$
u_{k}=u_{k}(\zeta)=\sum_{n=0}^{N} A_{2 k-1, n} \zeta^{n} / \sum_{n=0}^{N} A_{2 k, n} \zeta^{n}, \quad k=1,2,3,
$$

denote three rational functions of a complex variable $\zeta ; A_{k, n}, k=1,2, \cdots, 6$, $n=0,1,2, \cdots, N$, are complex numbers. Let

$$
\mathrm{X}=x_{1} \mathrm{i}_{1}+x_{2} \mathrm{i}_{2}+x_{3} \mathrm{i}_{3}
$$

represent the (variable) vector in three-dimensional Euclidean space; $x_{1}, x_{2}, x_{3}$

(12) This approach becomes particularly fruitful when we investigate complex functions $h\left(x_{1}, x_{2}, x_{3}\right)$ since in this case we can apply many results in the theory of value distribution of entire functions of a complex variable. 
are Cartesian coordinates in this space and $\mathrm{i}_{k}$ is a unit vector in the $x_{k}$ directions, $k=1,2,3$. We now form the expression $\left[\sum_{k=1}^{3}\left(x_{k}-u_{k}(\zeta)^{2}\right]^{1 / 2}\right.$. The term in brackets when multiplied by the product of the denominators of the $u_{k}$ 's is a polynomial, $P(\zeta, \mathrm{X})$, in $\zeta$ which depends upon $\mathrm{X}$. A formal computation yields

$$
\begin{aligned}
P(\zeta, \mathrm{X}) & =\left\{\left[\prod_{k=1}^{3}\left(\sum_{\mu=1}^{N} A_{2 k, \mu} \zeta^{\mu}\right)^{2}\right]\left[\sum_{k=1}^{3}\left(x_{k}-u_{k}(\zeta)\right)^{2}\right]\right\} \\
= & \sum_{k=1}^{3}\left[\sum_{n=0}^{N}\left(x_{k} A_{2 k, n}-A_{2 k-1, n}\right) \zeta^{n}\right]^{2} \cdot\left[\sum_{\mu=0}^{N} A_{2 k^{\prime}, \mu} \zeta^{\mu}\right]^{2} \cdot\left[\sum_{\sigma=0}^{N} A_{2 k^{\prime \prime}, \sigma} \zeta^{\sigma}\right]^{2} \\
= & \sum_{k=1}^{3} \sum\left(x_{k} A_{2 k, \nu}-A_{2 k-1, \nu}\right)\left(x_{k} A_{2 k, n}\right. \\
& \left.-A_{2 k-1, n}\right) A_{2 k^{\prime} \mu} A_{2 k^{\prime} m} A_{2 k^{\prime \prime}, \sigma} A_{2 k^{\prime \prime}, s} \zeta^{\nu+n+\mu+m+\sigma+\iota}
\end{aligned}
$$

Here $k^{\prime} \equiv k+1, k^{\prime \prime} \equiv k+2(\bmod 3), \sum_{\nu=0}^{N} \sum_{n=0}^{N} \sum_{\mu=0}^{N} \sum_{m=0}^{N} \sum_{\sigma=0}^{N} \sum_{s=0}^{N}$.

The algebraic function $[P(\zeta, \mathrm{X})]^{1 / 2}, \mathrm{X}$ fixed, defines a Riemann surface, $\Re(\mathrm{X})$. By

$$
e_{k}=e_{k}(\mathrm{X}), \quad n=0,1, \cdots, 6 N-1,
$$

we denote the branch points of $\Re(\mathrm{X})$. Clearly the $e_{k}(\mathrm{X})$ are the zeros of the polynomials $P(\zeta, \mathrm{X})$ (see (2.10)).

The points $\mathrm{X}$ of the space $\mathrm{r}$,

$$
\mathfrak{r}=\mathrm{E}\left[x_{1}^{2}+x_{2}^{2}+x_{3}^{2}<\infty\right],
$$

for which at least two branch points $e_{k}(\mathrm{X})$ coincide (although for other $\mathrm{X}$ differing from one another) will, in general, form an algebraic curve

$$
s_{1}=\mathrm{E}\left[P(\zeta, \mathrm{X})=0, P_{\zeta}(\zeta, \mathrm{X})=0\right], \quad P_{\zeta} \equiv \partial P / \partial \zeta .
$$

Remark 2.1. Eliminating $\zeta$ from both equations in the bracket of (2.6) we obtain an equation with complex coefficients whose arguments are $x_{1}, x_{2}, x_{3}$. Taking the real and imaginary parts of this equation, we obtain two real relations between $x_{1}, x_{2}, x_{3}$, which, in general, define a line. It may, however, happen that one of these equations vanishes identically $\left({ }^{13}\right)$, then (2.6) represents a surface unless the resulting equation is a line in $r$. (2.6) may, on the other hand, be only a point in $r$. (See the example given in $\$ 2$.)

We denote by $z_{2}$ the set

$$
\varepsilon_{2}=\mathrm{E}\left[\dot{b}_{0}(\mathrm{X}) \equiv \sum_{k=1}^{3}\left(x_{k} A_{2 k, N}-A_{2 k-1, N}\right)^{2} A_{2 k^{\prime}, N}^{2} A_{2 k^{\prime \prime}, N}^{2}=0\right]
$$

(13) If we were to consider our functions in the (six-dimensional) space of the three complex variables $x_{1}, x_{2}, x_{3}$, these exceptional cases would not appear. Since, however, we consider only the real (three-dimensional) space we must take into account that some exceptional cases do occur. In order to stress this fact, we shall, in the following, add the phrase "in general" when formulating results of this kind. 
and shall write

$$
\mathfrak{B}=\mathfrak{B}_{1}+\mathfrak{g}_{2} \text {. }
$$

Remark 2.2. If the $u_{k}$ are polynomials, then $\varepsilon_{2}$ is empty. If the $u_{k}$ are fractions of two polynomials then, in general, $\varepsilon_{2}$ is an intersection of two surfaces of second order. This intersection becomes a single point if all $A_{s, N}, N=1$, $2, \cdots, 6$, are real. We write

where

$$
\mathrm{E}(\mathrm{X}, \zeta)=\frac{1}{\left[\sum_{k=1}^{3}\left(x_{k}-u_{k}(\zeta)\right)^{2}\right]^{1 / 2}}=\prod_{k=1}^{3}\left(\sum_{\mu=1}^{N} A_{2 k, \mu} \zeta^{\mu}\right) /[P(\zeta, \mathrm{X})]^{1 / 2}
$$

$$
\begin{aligned}
P(\zeta, \mathrm{X}) & =b_{0}\left(\mathrm{X}_{\mathrm{i}}\right) \prod_{k=0}^{6 N-1}\left[\zeta-e_{\kappa}(\mathrm{X})\right], \\
b_{0}(\mathrm{X}) & =\sum_{k=1}^{3}\left(x_{k} A_{2 k, N}-A_{2 k-1, N}\right)^{2} \cdot A_{2 k^{\prime}, N}^{2} \cdot A_{2 k^{\prime \prime}, N}^{2},
\end{aligned}
$$

see (2.3).

We now define the operator $\mathbf{P}$ by

$$
\mathrm{P}\left[f, \mathfrak{R}, \mathrm{X} \in \mathfrak{u}\left(\mathrm{X}_{0}\right)\right]=\int_{\mathfrak{l}} \mathrm{E}(\mathrm{X}, \zeta) f(\zeta) d \zeta, \quad \mathrm{X} \in \mathfrak{U}\left(\mathrm{X}_{0}\right) .
$$

Here $\mathfrak{U}\left(\mathrm{X}_{0}\right)$ is a sufficiently small neighborhood of $\mathrm{X}_{0}$ and $f$ is an analytic function of $\zeta$ regular in a domain $\mathfrak{D}$, which domain includes the integration curve $\mathfrak{R} . \mathbb{R}$ is an oriented curve in the schlicht $\zeta$-plane, which is assumed to consist of finitely many regular arcs. The initial and end points of $\mathfrak{R}$ will be denoted by $\zeta_{0}$ and $\zeta_{1}$, respectively.

If $\mathfrak{R}$ is placed on the Riemann surface $\Re(\mathrm{X}), \mathrm{X} \in \mathfrak{r}-\mathfrak{B}$, then its initial point can be located either in one or in another sheet (except those cases where $\zeta_{0}$ coincides with one of the branch points $e_{\kappa}(\mathrm{X})$ of $\Re(\mathrm{X})$ ). If the curve $\mathfrak{R}$ does not intersect any branch point $e_{\kappa}(\mathrm{X})$ then the location of $\mathfrak{R}$ on $\Re(\mathrm{X})$ is uniquely determined by the choice of the sheet in which $\zeta_{0}$ lies. Thus:

Remark 2.3. For all values of $\mathrm{X}, \mathrm{X} \in \mathfrak{r}-\mathbb{8}$, for which $\&$ does not intersect any branch point $e_{\kappa}(\mathrm{X})$ of $\Re(\mathrm{X})$ the integral (2.11) is a two-valued function of X.

Definition 2.1. Let $\mathbf{E}$ be a fixed function in troduced in (2.9). If $\mathbb{R}$ ranges over all possible admissible curves and $f(\zeta)$ ranges over all analytic functions satisfying the above conditions, then the totality of functions represented by the integral (2.11) forms a class of harmonic functions, which class will be denoted by $\mathfrak{H C}(\mathbf{E})$.

If we restrict the set of admissible integration curves $\mathfrak{L}$, requiring that their initial and end points are two fixed points, say $\zeta_{0}$ and $\zeta_{1}$, then the in- 
tegral (2.11) will range over a subclass of functions $\mathfrak{H C}(\mathbb{E})$, which subclass will be denoted by $\mathcal{S}\left(\mathbf{E}, \zeta_{0}, \zeta_{1}\right)$.

2. Examples. 1. Let

$$
u_{k}(\zeta)=a_{k}+b_{k} \zeta, \quad k=1,2,3
$$

Then

$$
P(\zeta, \mathrm{X})=\sum_{k=1}^{3}\left(x_{k}-a_{k}-b_{k} \zeta\right)^{2} .
$$

The line 8 (see (2.8), (2.6), (2.7)) is in this case the intersection of two surfaces of the second order, namely of

$$
\begin{aligned}
& \sum_{\nu=1}^{3}\left\{x_{\nu}^{2} \sum_{k=1}^{2}(-1)^{k+1}\left(\alpha_{\nu^{\prime}}^{(k) 2}+\alpha_{\nu^{\prime \prime}}^{(k) 2}+\alpha_{\nu^{\prime}}^{(k)} \alpha_{\nu^{\prime \prime}}^{(k)}\right)\right. \\
& +x_{\nu^{\prime}} x_{\nu^{\prime}} \sum_{k=1}^{2}(-1)^{k+1} \alpha_{\nu}^{(\alpha)}\left(\alpha_{\nu}^{(k)}-\alpha_{\nu^{\prime}}^{(k)}-\alpha_{\nu^{\prime \prime}}^{(k)}\right) \\
& -x, \sum_{\alpha=1}^{2}(-1)^{\kappa}\left[A_{\nu}^{(\kappa)}\left(\alpha_{\nu^{\prime}}^{(\kappa)}+\alpha_{\nu^{\prime \prime}}^{(\kappa)}\right)-A_{\nu^{\prime}}^{(\kappa)}+\alpha_{\nu^{\prime}}^{(\kappa)}-A_{\nu^{\prime \prime}}^{(\kappa)} \alpha_{\nu^{\prime \prime}}^{(\kappa)}\right] \\
& \left.-\left(A_{\nu}^{(1)^{2}}-A_{\nu}^{(2)^{2}}\right) / 2\right\}=0
\end{aligned}
$$

and of

$$
\begin{aligned}
\sum_{v=1}^{3} x_{\nu}^{2} & \sum_{k=1}^{3} \sum_{s=1}^{3} \alpha_{k}^{(1)} \alpha_{s}^{(2)} \\
& -\sum_{v=1}^{3} \sum_{k=1}^{3} x_{v} x_{k} \sum_{s=1}^{3}\left(\alpha_{\nu}^{(2)} \alpha_{s}^{(1)}-\alpha_{\nu}^{(1)} \alpha_{s}^{(2)}\right) \sum_{\nu=1}^{3} x_{\nu} \sum_{k=1}^{3}\left(A_{\nu}^{(1)} \alpha_{k}^{(2)}+A_{\nu}^{(2)} \alpha_{k}^{(1)}\right) \\
& -\sum_{k=1}^{3} x_{k} \sum_{v=1}^{3}\left(\alpha_{\nu}^{(1)} A_{k}^{(2)}+\alpha_{\nu}^{(2)} A_{k}^{(1)}\right)+A_{\nu}^{(1)} A_{\nu}^{(2)}=0
\end{aligned}
$$

where $\nu^{\prime} \equiv \nu+1, \nu^{\prime \prime} \equiv \nu+2(\bmod 3)$,

2. Let

$$
b_{k}=\alpha_{k}^{(1)}+i \alpha_{k}^{(2)} \text { and } A_{\nu}^{(1)}+i A_{\nu}^{(2)}=-a_{\nu} \sum_{k=1}^{8} b_{k}+b_{\nu} \sum_{k=1}^{3} a_{k} .
$$

$$
u_{k}=v_{k}(\zeta),
$$$$
0 \leqq \zeta \leqq 1,
$$

be three real rational functions. Then 8 becomes the curve $\mathrm{E}\left[x_{k}=v_{k}(\zeta)\right.$, $0 \leqq \zeta \leqq 1$ ]. Assuming that $f(\zeta)$ is real for $0 \leqq \zeta \leqq 1$, the expression (2.11) represents the Newtonian potential of linear charge distribution along the above line.

3. $u_{k}=A_{k 2} \zeta^{2}$. Then 


$$
\begin{aligned}
& P(\zeta, \mathrm{X})=\sum_{k=1}^{3}\left(x_{k}-A_{k 2} \zeta^{2}\right)=\sum_{k=1}^{3} x_{k}^{2}-2 \zeta^{2} \sum_{k=1}^{3} A_{k 2} x_{k}+\zeta^{4} \sum_{k=1}^{3} A_{k 2}^{2}, \\
& P_{0}(\zeta, \mathrm{X})=-4 \zeta \sum_{k=1}^{3} A_{k 2} x_{k}+4 \zeta^{3} \sum_{k=1}^{3} A_{k 2}^{2}, \\
& 8_{1}=\mathrm{E}\left[\sum_{k=1}^{3} x_{k}^{2}-\frac{\left(\sum_{k=1}^{3} A_{k 2} x_{k}\right)^{2}}{\sum_{k=1} A_{k 2}^{2}}=0\right]=\mathrm{E}\left[\sum_{k=1}^{3} x_{k}^{2} \sum_{k=1}^{3} A_{k 2}^{2}-\left(\sum_{k=1}^{3} A_{k 2} x_{k}\right)^{2}\right] \\
& =\mathrm{E}\left[x_{1}^{2}\left(A_{22}^{2}+A_{32}^{2}\right)+x_{2}^{2}\left(A_{12}^{2}+A_{32}^{2}\right)+x_{3}^{2}\left(A_{22}^{2}+A_{32}^{2}\right)\right. \\
& \left.-2 x_{1} x_{2} A_{12} A_{22}-2 x_{1} x_{3} A_{12} A_{32}-2 x_{2} x_{3} A_{22} A_{32}=0\right] .
\end{aligned}
$$

$\xi_{2}$ is empty in this case.

We obtain for the $e_{k}(\mathrm{X})$

$$
e_{k}(\mathrm{X})= \pm\left[\frac{\sum_{k=1}^{3} A_{k 2} x_{k} \pm\left(\left(\sum_{k=1}^{3} A_{k 2} x_{k}\right)^{2}-\sum_{k=1}^{3} A_{k 2}^{2} \sum_{k=1}^{3} x_{k}^{2}\right)^{1 / 2}}{\sum_{k=1}^{3} A_{k 2}^{2}}\right]^{1 / 2} .
$$

3. The domain, $\mathfrak{a}$, in which the integral (2.16) exists. The function $h(\mathrm{X})$ is defined by $(2.11)$ in a sufficiently small neighborhood $\mathfrak{u}\left(\mathrm{X}_{0}\right)$ of $\mathrm{X}_{0}$. The first problem which arises is to determine the domain, $\mathfrak{a}$, of $\mathfrak{r}-\mathfrak{b}$ for which the integral

exists.

$$
\int_{\mathrm{g}} \mathrm{E}(\mathrm{X}, \zeta) f(\zeta) d \zeta=\int_{\zeta_{0}}^{\zeta_{1}} \frac{\left[\prod_{k=1}^{3}\left(\sum_{\mu=0}^{N} A_{2 k, \mu} \zeta^{\mu}\right)\right] f(\zeta)}{\left[b_{0}(X) \prod_{k=0}^{6 N-1}\left(\zeta-e_{k}(\mathrm{X})\right)\right]^{1 / 2}} d \zeta
$$

To every point $\zeta$, say $\zeta=\zeta_{0}\left({ }^{14}\right)$, there corresponds in the $\mathrm{X}$ space a circle $\mathfrak{P}\left(\zeta_{0}\right)$,

$$
\mathfrak{P}\left(\zeta_{0}\right)=\mathrm{E}\left[P\left(\zeta_{0}, \mathrm{X}\right)=0\right],
$$

which is the intersection of the sphere

$$
\begin{aligned}
& S\left(\zeta_{0}\right)=\mathrm{E}\left[\sum_{k=1}^{3}\left(x_{k}-v_{k}\left(\zeta_{0}\right)^{2}\right)-\sum_{k=1}^{3} w_{k}^{2}\left(\zeta_{0}\right)=0\right], \\
& u_{k}\left(\zeta_{0}\right)=v_{k}\left(\zeta_{0}\right)+i w_{k}\left(\zeta_{0}\right),
\end{aligned}
$$

(14) Note that $\zeta_{0}$ is sometimes employed to denote an arbitrary point of the $\zeta$-plane, sometimes the initial point of $\mathbb{R}+\mathrm{L}$ and sometimes an interior point of $\mathbb{R}$. This should cause no confusion as it will be clear from the context which is meant. 
of radius $\rho\left(\zeta_{0}\right)=\left[\sum_{k=1}^{3} w_{k}^{2}\left(\zeta_{0}\right)\right]^{1 / 2}$ and center at $\left[v_{1}\left(\zeta_{0}\right), v_{2}\left(\zeta_{0}\right), v_{3}\left(\zeta_{0}\right)\right]$, and of the plane

$$
\mathfrak{A}\left(\zeta_{0}\right)=\mathrm{E}\left[\sum_{k=1}^{3}\left(x_{k}-v_{k}\left(\zeta_{0}\right)\right) w_{k}\left(\zeta_{0}\right)=0\right]
$$

passing through the above center. We note that the function $\mathrm{E}\left(\mathrm{X}, \zeta_{0}\right)$ (see (2.9)) is regular at every point of $\mathfrak{r}-\boldsymbol{z}$ except on $\mathfrak{B}\left(\zeta_{0}\right)$ where it becomes infinite.

THEOREM 2.1. Let $f(\zeta)$ be a regular function of $\zeta$ at every point of $\mathfrak{Q}+\mathrm{L}\left({ }^{15}\right)$; then the integral (2.16) represents throughout

$$
\mathfrak{a}=\mathfrak{r}-\mathfrak{z}-\Im, \quad \Im=\left[\sum \mathfrak{B}(\zeta), \zeta \in \mathfrak{R}+\mathrm{L}\right],
$$

two functions, both of which are defined at every point of $\mathfrak{a}$.

Proof. 1. When $\zeta$ ranges over $\mathfrak{R}$, the expression $\mathrm{E}(\mathrm{X}, \zeta)$ in $(2.9)$ becomes infinite only at those points $\mathrm{X}$ which belong to $\Im$. For other values of $\mathrm{X}$, $\mathrm{X} \in \mathfrak{r}-\mathrm{z}, \mathrm{E}(\mathrm{X}, \zeta)$ is a regular function of $\zeta$, and therefore the integral (2.16) exists at every point $\mathrm{X}$ of the domain (2.20).

2. We shall now prove that (2.16) is two-valued. According to Remark 2.3 it will suffice to show that for all points $\mathrm{X}$ of $\mathfrak{a}$ the integration curve $\mathfrak{l}$ does not intersect any branch points $e_{\kappa}(\mathrm{X}), \kappa=0, \cdots, 6 N-1$, of $\Re(\mathrm{X})$.

$\mathrm{E}(\mathrm{X}, \zeta)$ becomes infinite only at those points $\mathrm{X}$ for which the denominator

$$
P(\zeta, \mathrm{X})=b_{0}(\mathrm{X})\left[\prod_{k=0}^{6 N-1}\left(\zeta-e_{k}(\mathrm{X})\right)\right]
$$

(see (2.9) and (2.10)) vanishes. But if some point $\zeta$, say $\zeta_{0}$, of $\mathfrak{Q}$ coincides with a branch point $e_{\kappa}(\mathrm{X})$, then the corresponding $\mathrm{X}$ lies according to (2.17) on $\mathfrak{B}\left(\zeta_{0}\right)$, and therefore by $(2.20)$ on $\Im$. Consequently $\mathrm{X}$ does not belong to $\mathfrak{a}$.

According to whether we locate the initial point in one or another sheet of $\Re(\mathrm{X})$ we obtain (in general) two different values for (2.16).

From the above considerations it follows immediately:

COROLlaRy 2.1. In every connected part of a, every branch of the integral (2.16) represents a harmonic function of $x_{1}, x_{2}, x_{3}$.

4. The discontinuity surface $\Im$. In the following we shall denote by $\delta$ the totality of those points $\zeta$ of the schlicht $\zeta$ plane for which

$$
\rho(\zeta)=\left[\sum_{k=1}^{3} w_{k}^{2}(\zeta)\right]^{1 / 2}
$$

(15) We denote the boundary of the manifold under consider by the corresponding Roman letter, for example $\mathfrak{L}+L, \mathfrak{g}+g, \mathfrak{p}+\mathrm{p}$ denote the curves $\mathfrak{R}, \mathfrak{g}, \mathfrak{p}$, together with their boundary points. 
vanishes. In this case the circle $\mathfrak{B}(\zeta)$ (see (2.17)) degenerates to a point.

Remark 2.4. In the case of Example 2, the curve $\mathfrak{R}$ consists of points of $\delta$, and $\Im=\left[\sum \mathfrak{B}(\zeta), \zeta \in \mathbb{R}\right]$ degenerates to a curve, which represents the line of (linear) charge distribution.

We shall assume in the following in this section that $R \cap \delta=0$.

In this case every $\mathfrak{P}(\zeta)$ divides the plane $\mathfrak{A}(\zeta)$ into two parts (see Fig. 2),

$$
\mathfrak{u}_{\kappa}(\zeta)=\mathrm{E}\left\{(-1)^{\kappa}\left[\sum_{k=1}^{3}\left(x_{k}-v_{k}(\zeta)\right)^{2}-w_{k}^{2}(\zeta)\right]>0\right\}, \quad \kappa=1,2 .
$$

The points of $\mathfrak{u}_{2}(\zeta)$ can be connected with $\mathrm{X}=\infty$ by a curve lying in $\mathfrak{A}(\zeta)$ which does not intersect $\mathfrak{P}(\zeta)$; in order to connect a point of $\mathfrak{u}_{1}(\zeta)$ with $\mathrm{X}=\infty$ we have to cut $\mathfrak{B}(\zeta)$ once or in general an odd number of times.

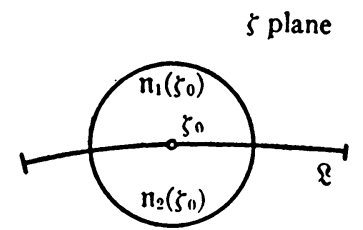

FIG. 1. The neighborhood $\mathfrak{n}(\zeta)$ of a point $\zeta_{0}$.

Remark 2.5. If through a point $\mathrm{X}$, say $\mathrm{X}=\mathrm{X}_{1}$, there passes only one $\mathfrak{B}(\zeta)$, $\zeta \in \mathbb{R}$, then the (sufficiently small) neighborhood $\mathfrak{u}\left(\mathrm{X}_{1}\right)$ of $\mathrm{X}_{1}$ is divided into two parts $\mathfrak{U}_{\kappa}\left(\mathrm{X}_{1}\right), \kappa=1,2$. $\left(\mathfrak{U}_{\kappa}\left(\mathrm{X}_{1}\right)\right.$ is assumed to include $\mathfrak{u}_{\kappa}\left[e_{\nu}\left(\mathrm{X}_{1}\right)\right], e_{\nu}\left(\mathrm{X}_{1}\right) \in \mathfrak{l}$, $\kappa=1,2$.) Each of the neighborhoods $\mathfrak{U}_{\kappa}\left(X_{1}\right)$ is connected. Therefore each branch of the integral (2.11) yields two harmonic functions, say $h_{1}(\mathrm{X})$ and $h_{2}(\mathrm{X})$, according to whether the point $\mathrm{X}_{0}$ lies in $\mathfrak{U}_{1}\left(\mathrm{X}_{1}\right)$ or $\mathfrak{U}_{2}\left(\mathrm{X}_{1}\right)$.

Definition 2.2. Let $\zeta_{0}$ be a point of $\mathfrak{R}$, and $\mathfrak{n}\left(\zeta_{0}\right)$ a sufficiently small neighborhood of $\zeta_{0}$. $\mathfrak{R}$ being a smooth curve divides $\mathfrak{n}\left(\zeta_{0}\right)$ into two parts $\mathfrak{n}_{\mathfrak{k}}\left(\zeta_{0}\right)$, $\kappa=1$, 2. See Fig. 1. Suppose that through the point $\mathrm{X}_{0}$ of $\Im=\left[\sum \Re(\zeta), \zeta \in \mathbb{R}\right]$, there passes only one circle $\mathfrak{P}(\zeta), \zeta \in \mathfrak{R}$, say $\mathfrak{B}\left(\zeta_{0}\right)$. See Fig. 2. If to every $\zeta \in \mathfrak{n}_{\nu}\left(\zeta_{0}\right)$, we have $\left({ }^{16}\right)$

$$
[\mathfrak{B}(\zeta)] \cap\left[\mathfrak{U}\left(\mathrm{X}_{0}\right)\right] \in \mathfrak{u}_{\mu}\left(\mathrm{X}_{0}\right), \quad \quad \mu=1,2,
$$

and, vice versa, to every point $\mathrm{X} \in \mathfrak{U}_{\mu}\left(\mathrm{X}_{0}\right)$ the corresponding point $\zeta=e_{x}(\mathrm{X})$ (which lies in the neighborhood $\mathfrak{n}\left(\zeta_{0}\right)$ ) lies in $\mathfrak{n}_{\nu}\left(\zeta_{0}\right), \nu=1,2$, the point will be said to be a regular point of $\Im$.

In the case where several circles $\mathfrak{B}\left(\zeta^{(\nu)}\right), \nu=0,1, \cdots, \sigma, \zeta^{(\nu)} \in \mathfrak{R}$, pass through $\mathrm{X}_{0}, \mathrm{X}_{0}$ will be said to be a regular point of $\Im=\left[\sum \mathfrak{B}(\zeta), \zeta \in \mathbb{R}\right]$ if it is possible to decompose $\mathfrak{R}$ into a finite number of parts, $\mathscr{R}=\sum_{\nu=1}^{\sigma} \mathbb{R}_{\nu}$, such that

(18) Note that the above statement has to hold for $\nu=1$ as well as for $\nu=2$, and that the neighborhood $\mathfrak{n}_{1}\left(\zeta_{0}\right)$ may be mapped into either the neighborhood $\mathfrak{u}_{1}\left(\mathrm{X}_{0}\right)$ or $\mathfrak{U}_{2}\left(\mathrm{X}_{0}\right)$. In the former case $\mathfrak{n}_{2}\left(\zeta_{0}\right)$ will map into $\mathfrak{U}_{2}\left(\mathrm{X}_{0}\right)$ while in the latter it will map into $\mathfrak{U}_{1}\left(\mathrm{X}_{0}\right)$. 
In $\S 8$ sufficient conditions in order that a point $\mathrm{X}$ be a regular point of $\Im$ will be derived.

If several $\mathfrak{B}(\zeta), \zeta \in \mathbb{R}$, pass through the point $\mathrm{X}$, then the neighborhood $\mathfrak{u}(\mathrm{X})$ will, in general, be divided into finitely many connected neighborhoods $\mathfrak{U}_{\kappa}(\mathrm{X}), \kappa=1,2, \cdots, s$. Each branch of the integral (2.16) defines in each $\mathfrak{U}_{\kappa}(\mathrm{X})$ a regular harmonic function $h_{\kappa}(\mathrm{X}), \kappa=1,2, \cdots, s$.

5. The proof that each of the functions $h_{\kappa}(\mathrm{X})$ is regular at every regular point $\mathrm{X}_{1}$ of $\Im-\mathfrak{B}\left(\zeta_{0}\right)-\mathfrak{B}\left(\zeta_{1}\right)$.

THEOREM 2.2. Let $f(\zeta)$ be regular on $R+\mathrm{L}$, let $(\Omega+L) \cap \delta=0$ and let the point $\mathrm{X}_{1}, \mathrm{X}_{1} \in \mathfrak{r}-\mathfrak{B}$, be a regular point of $\mathfrak{\Im}-\mathfrak{B}\left(\zeta_{0}\right)-\mathfrak{B}\left(\zeta_{1}\right)$.

The functions $h_{\kappa}(\mathrm{X})$ defined by (2.16) in $\mathfrak{U}_{\kappa}, \kappa=1,2, \cdots, s$, are regular at the point $\mathrm{X}_{1}$.

Proof. Since $X_{1} \in \mathfrak{F}-\mathfrak{B}\left(\zeta_{0}\right)-\mathfrak{B}\left(\zeta_{1}\right)=\mathrm{E}\left[\sum \mathfrak{P}(\zeta), \zeta \in \mathbb{R}\right]$ there must exist a number of points, say $\zeta^{(v)}, \nu=1,2, \cdots, \sigma$ on $\mathbb{R}$, such that $P\left(\zeta^{(v)}, \mathrm{X}_{1}\right)=0$. We decompose $\mathbb{R}$ into $\sigma$ parts, $\mathbb{R}_{\nu}, \mathbb{R}=\sum_{\nu=1}^{\sigma} \mathbb{R}_{\nu}, \mathbb{R}_{\nu} \cap \mathfrak{R}_{\mu}=0$, such that in every $\mathbb{R}_{\mu}$ there lies only one $\zeta^{(v)}$, say $\zeta^{(\mu)} \cdot h(\mathrm{X})$ given by (2.16) may be represented in the form

$$
h(\mathrm{X})=\sum_{\nu=1}^{\sigma} h^{(\nu)}(\mathrm{X}), \quad h^{(\nu)}(\mathrm{X})=\int_{\mathfrak{\Omega}_{\nu}} \mathbf{E}(\mathrm{X}, \zeta) f(\zeta) d \zeta .
$$

We now consider a single function

$$
h^{(\nu)}(\mathbf{X})=\int_{\Omega_{\nu}} \mathbf{E}(\mathbf{X}, \zeta) f(\zeta) d \zeta,
$$

at the point $\mathrm{X}_{1}$. For $\zeta \in \Omega_{\text {, the }}$ there exists only one point $\zeta=\zeta^{(\nu)}$ such that $\mathfrak{P}\left(\zeta^{(v)}\right)$ passes through $\mathrm{X}_{1}$. The surface $\Im_{\nu}=\mathrm{E}\left[\sum \mathfrak{B}(\zeta), \zeta \in \mathfrak{R}_{\nu}\right]$ divides a sufficiently small neighborhood $\mathfrak{u}\left(\mathrm{X}_{1}\right)$ (see Fig. 2) into two simply connected parts $\mathfrak{U}_{\kappa}\left(\mathrm{X}_{1}\right), \kappa=1,2$, in which we define two functions.

$$
h_{\kappa}^{(\nu)}(\mathrm{X})=\int_{\mathbb{\Omega}_{\nu}} \mathrm{E}(\mathrm{X}, \zeta) f(\zeta) d \zeta, \quad \mathrm{X} \in \mathfrak{U}_{\kappa}\left(\mathrm{X}_{1}\right), \kappa=1,2 .
$$

(Clearly it may occur that both $h_{\kappa}^{(\nu)}(\mathrm{X}), \kappa=1,2$, are function elements of the same function.)

Let

$$
\delta=\min \left[\left|e_{\kappa}\left(\mathrm{X}_{1}\right)-e_{\sigma}\left(\mathrm{X}_{1}\right)\right|, \kappa \neq \sigma ; \sigma, \quad \kappa=0,1,2, \cdots, 6 N-1\right]
$$

and let $\epsilon<\delta / 2$ be a sufficiently small positive quantity. We introduce now instead of $\mathfrak{R}_{\nu}$ a new (smooth) curve $\mathfrak{R}_{\nu}^{*}$ which possesses the same end points $\alpha$ and $\beta$, but we replace the interior segment $a \zeta^{(\nu)} b$ by $a m b$, which lies in $\mathfrak{n}_{2}\left(\zeta^{(\nu)}\right)$ and has the property that the distance of every point of $a m b$ from $\zeta^{(v)}$ is larger than $\epsilon / 2$ but smaller than $\epsilon$. (See Fig. 3.) $X_{1}$ does not lie on $\Im_{\nu}^{*}=\mathrm{E}\left[\sum \mathfrak{B}(\zeta), \zeta \in \mathbb{R}_{\nu}^{*}\right]$. For, by assumption, no zeros of $P\left(\zeta, \mathrm{X}_{1}\right)$ lie on $\alpha a$ or $b \beta$. Were a zero, say $\zeta^{(s)}$ of $P\left(\zeta, \mathrm{X}_{1}\right)$, situated on $a m b$ then we should have 


$$
\left|e_{s}\left(\mathrm{X}_{1}\right)-e_{\nu}(\mathrm{X})\right|<\epsilon<\delta
$$

in contradiction with (2.28).

Let $\mathrm{X}_{0}$ of $(2.11)$ be a point of $\mathfrak{U}_{1}\left(\mathrm{X}_{1}\right)$. We shall show that in this case the function $h_{1}^{(\nu)}(\mathrm{X})$ obtained by applying the operator $(2.11)$ (where $\mathbb{R}=\mathbb{R}_{\nu}$ ) re-

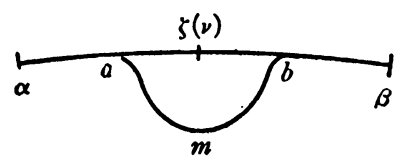

FIG. 3. The curves $\mathfrak{R}$ and $\mathfrak{l}^{*}$.

mains unchanged when the integration curve $\mathbb{R}_{\nu}=\alpha a+a \zeta^{(\nu)} b+b \beta$ is replaced by $\mathfrak{R}_{\nu}^{*}=\alpha a+a m b+b \beta$, that is, that

$$
h_{1}^{(\nu)}(\mathbf{X})=\int_{\mathbf{g}_{\nu}} \mathbf{E}(\mathbf{X}, \zeta) f(\zeta) d \zeta=\int_{\mathbf{R}_{\nu}^{*}} \mathbf{E}(\mathbf{X}, \zeta) f(\zeta) d \zeta
$$

holds.

Indeed, since $\mathrm{X}_{0} \in \mathfrak{U}_{1}\left(\mathrm{X}_{1}\right)$ and $\mathrm{X}_{1}$ is a regular point of $\Im$, no zero of $P(\zeta, \mathrm{X})$ lies in $\mathfrak{n}_{1}\left(\zeta^{(\nu)}\right)$. Therefore, when by a continuous and one-to-one transformation we deform $\mathfrak{R}_{\nu}{ }^{*}$ into $\mathfrak{R}_{\nu}$, that is, when the arc $a m b$ sweeps over the area bounded by $a \zeta^{(v)} b m a$, we do not encounter any singularity of $\mathrm{E}\left(\mathrm{X}_{0}, \zeta\right) f(\zeta)$; for, by assumption $f(\zeta)$ is regular in the neighborhood $n\left(\zeta^{(\nu)}\right)$ of $\zeta^{(v)}$ and the singularities of $\mathrm{E}\left(\mathrm{X}_{0}, \zeta\right)$ are zeros of $P\left(\zeta, \mathrm{X}_{0}\right)$ which lie in $\mathfrak{n}_{1}\left(\zeta^{(\nu)}\right)$, while the above domain (bounded by $a \zeta^{(\nu)} b m a$ ) lies in $\mathfrak{n}_{2}\left(\zeta^{(\nu)}\right)$. On the other hand $\mathrm{E}\left(\mathrm{X}_{1}, \zeta\right), \zeta \in \mathbb{R}_{\nu}^{*}$, is not infinite and the second integral of $(2.30)$ is regular at $X=X_{1}$. Since in every neighborhood of $\mathrm{X}_{1}$ there are points of $\mathfrak{U}_{1}\left(\mathrm{X}_{1}\right)$, the right-hand integral of (2.30) is the analytic continuation of $h_{1}{ }^{(v)}(\mathrm{X})$ into the point $\mathrm{X}_{1}$.

6. The saltus of the integral (2.16) on passing a discontinuity surface $\mathfrak{F} \mathfrak{P}\left(\zeta_{0}\right)-\mathfrak{P}\left(\zeta_{1}\right)$. Let $\mathrm{X}_{0}$ be a regular point of $\mathfrak{Y}-\mathfrak{B}\left(\zeta_{0}\right)-\mathfrak{B}\left(\zeta_{1}\right)$. As was indicated in $\$ 4$ the neighborhood $\mathfrak{u}\left(\mathrm{X}_{0}\right)$ is subdivided by $\Im$ into finitely many connected neighborhoods $\mathfrak{U}_{\kappa}\left(\mathrm{X}_{0}\right), \kappa=1,2, \cdots, s$. Each branch of (2.16) defines in every neighborhood $\mathfrak{U}_{\kappa}\left(\mathrm{X}_{0}\right)$ a harmonic function $h_{\nu}(\mathrm{X})$. If one passes from $\mathfrak{U}_{\kappa}\left(\mathrm{X}_{0}\right)$ into $\mathfrak{U}_{\sigma}\left(\mathrm{X}_{0}\right)$ through the point $\mathrm{X}_{0}$ the integral (2.16) undergoes the saltus

$$
h_{\sigma}\left(\mathrm{X}_{0}\right)-h_{\kappa}\left(\mathrm{X}_{0}\right)
$$

Theorem 2.3. The saltus (2.31) of the integral (2.16) on passing through a regular point $\mathrm{X}_{0}$ from $\mathfrak{U}_{\kappa}\left(\mathrm{X}_{0}\right)$ to $\mathfrak{U}_{\sigma}\left(\mathrm{X}_{0}\right)$ equals either

$$
+\int_{\zeta_{1,1}}^{\zeta_{1,2}} \frac{\left[\prod_{k=1}^{3}\left(\sum_{\mu=0}^{N} A_{2 k, \mu} \zeta^{\mu}\right)\right] f(\zeta) d \zeta}{\left[b_{0}\left(\mathrm{X}_{0}\right) \prod_{k=0}^{6 N-1}\left(\zeta-e_{k}\left(\mathrm{X}_{0}\right)\right)\right]^{1 / 2}}
$$


taken over some conveniently chosen path, or some combination of such integrals with integral coefficients. Here $\zeta_{1,1}=\left[\zeta_{1},+\left(P\left(\zeta_{1}, \mathrm{X}_{0}\right)\right)^{1 / 2}\right]$ and $\zeta_{1,2}$ $=\left[\zeta_{1},+\left(P\left(\zeta_{1}, \mathrm{X}_{0}\right)\right)^{1 / 2}\right]$, that is, the points which are situated in the first and second sheet of $\Re\left(\mathrm{X}_{0}\right)$ and whose $\zeta$ coordinate equals $\zeta_{1}$.

Proof. According to (2.20) through the point $\mathrm{X}_{0}$ passes one or several circles $\mathfrak{P}\left(\zeta^{(v)}\right), \zeta^{(v)} \in \mathbb{R}$, since $\mathrm{X}_{0} \in \Im$. We assume at first that only one circle, say $\mathfrak{B}\left(\zeta^{(0)}\right)$, goes through $\mathrm{X}_{0}$. According to the considerations of $\S 3$, see (2.17) and (2.21), one branch point of $\Re\left(\mathrm{X}_{0}\right)$, say $e_{k}\left(\mathrm{X}_{0}\right)$, must lie on $\mathfrak{R}$, and according to (2.21) we have $e_{k}\left(\mathrm{X}_{0}\right)=\zeta^{(0)}$ (see Fig. $4 \mathrm{~b}$ ). Let $\mathfrak{u}\left(\mathrm{X}_{0}\right)$ be a sufficiently small neighborhood of $X_{0}$. As indicated before, see $\S 4, \Im$ divides $\mathfrak{u}\left(X_{0}\right)$ into two connected parts, say $\mathfrak{U}_{1}\left(\mathrm{X}_{0}\right)$ and $\mathfrak{U}_{2}\left(\mathrm{X}_{0}\right)$. Further let $\mathrm{X}_{1}$ be a point of $\mathfrak{U}_{1}\left(\mathrm{X}_{0}\right)$; through $\mathrm{X}_{1}$ passes some circle $\mathfrak{B}(\zeta)$, say $\mathfrak{B}\left(\zeta^{(1)}\right)=\mathrm{E}\left[P\left(\zeta^{(1)}, \mathrm{X}_{1}\right)=0\right]$.

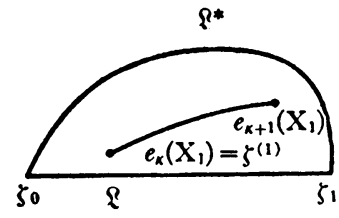

A

FIg. 4a. Riemann's surface $\Re\left(\mathrm{X}_{1}\right)$.

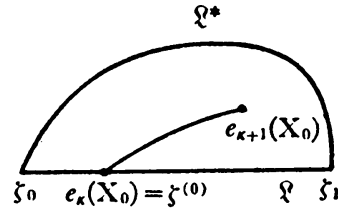

B

Fig. 4b. Riemann's surface $\Re\left(\mathrm{X}_{0}\right)$.

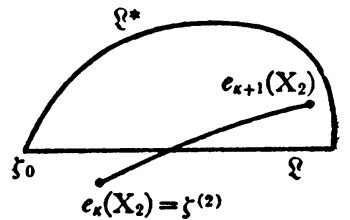

$\mathrm{C}$

FIG. 4c. Riemann's surface $\Re\left(\mathrm{X}_{2}\right)$.

From (2.17) and (2.21), it follows that $\zeta^{(1)}$ equals some branchpoint of Riemann's surface $\Re\left(\mathrm{X}_{1}\right)$, say (see Fig. $4 \mathrm{a}$ )

$$
\zeta^{(1)}=e_{\rho}\left(\mathrm{X}_{1}\right) \text {. }
$$

Since the distance $\left|e_{\mu}\left(\mathrm{X}_{0}\right)-e_{k}\left(\mathrm{X}_{0}\right)\right|, \mu \neq \kappa$, is larger than a fixed constant and since the zeros, $e_{\sigma}(\mathrm{X})$, of $P(\zeta, \mathrm{X})=0$ vary continuously with $\mathrm{X}, \rho$ must be equal to $\kappa$, that is, we must have

$$
\zeta^{(1)}=e_{k}\left(\mathrm{X}_{1}\right)
$$

if the point $\mathrm{X}_{1}$ is near enough to $\mathrm{X}_{0}$ and if $\zeta^{(1)}$ belongs to a sufficiently small neighborhood $\mathfrak{n}\left(\zeta^{(0)}\right)$ of $\zeta^{(0)}$. Similarly we choose a point $\mathrm{X}_{2} \in \mathfrak{H}_{2}\left(\mathrm{X}_{0}\right)$ which must lie on the circle $E\left[P\left(\zeta^{(2)}, \mathrm{X}_{2}\right)=0\right]$ such that (see Fig. 4c)

$$
\zeta^{(2)}=e_{\kappa}\left(\mathrm{X}_{2}\right) \text {. }
$$

According to our assumption only one $\mathfrak{B}\left(\zeta^{(0)}\right), \zeta^{(0)} \in \mathfrak{R}$, goes through $\mathrm{X}_{0}$. The neighborhood $\mathfrak{n}\left(\zeta^{(0)}\right)$ is divided by $\mathfrak{R}$ into two connected parts $\mathfrak{n}_{1}\left(\zeta^{(0)}\right)$ and $\mathfrak{n}_{2}\left(\zeta^{(0)}\right)$. Since $X_{0}$ is a regular point of $\Im$, and since $X_{1}$ lies on one and $\mathrm{X}_{2}$ on another side of $\Im, \zeta^{(1)}$ must lie in one part, say $\mathfrak{n}_{1}\left(\zeta^{(0)}\right)$, and $\zeta^{(2)}$ in another part, say $\mathfrak{n}_{2}\left(\zeta^{(0)}\right)$ of $\mathfrak{n}\left(\zeta^{(0)}\right)$. Since $X_{1}$ and $X_{2}$ belong to $\mathfrak{a}$, they are outside of $\Im$, and $e_{k}\left(\mathrm{X}_{s}\right), s=1,2$, can not lie on $\mathfrak{R}$.

Let $\left[e_{k}(\mathrm{X}), e_{\kappa+1}(\mathrm{X})\right], \mathrm{X} \in \mathfrak{U}\left(\mathrm{X}_{0}\right)$, be a cut of the Riemann surface $\Re(\mathrm{X})$ 
along which one sheet of $\Re(\mathrm{X})$ is connected with another sheet. Since we have assumed that only one circle $\mathfrak{P}(\zeta)$ passes through $\mathrm{X}, \boldsymbol{e}_{\kappa+1}(\mathrm{X}), \mathrm{X} \in \mathfrak{U}\left(\mathrm{X}_{0}\right)$, does not lie on $\mathbb{R}$. Since further $e_{k}\left(\mathrm{X}_{1}\right)$ lies on one side of $\mathbb{R}$ and $e_{k}\left(\mathrm{X}_{2}\right)$ on another side of $\mathfrak{R}$, we can assume that $\mathfrak{R}$ does not intersect $\left[e_{x}\left(\mathrm{X}_{1}\right), e_{x+1}\left(\mathrm{X}_{1}\right)\right]$ and cuts $\left[e_{k}\left(\mathrm{X}_{2}\right), e_{x+1}\left(\mathrm{X}_{2}\right)\right]$ only once.

Now let $\Omega^{*}$ be a new curve which is chosen in such a manner that the point $e_{\kappa}\left(\mathrm{X}_{1}\right)$ lies inside the domain bounded (in the schlicht plane) by $\mathfrak{R}+\mathfrak{R}^{*}$, and $e_{k}\left(\mathrm{X}_{2}\right)$ lies outside $\mathfrak{R}+\mathfrak{R}^{*}$. Note that on $\Re\left(\mathrm{X}_{2}\right), \mathfrak{R}+\mathfrak{R}^{*}$ is not a closed curve since the end point

$$
\zeta_{1,1}=\left[\zeta_{1},+\left(P\left(\zeta_{1}, \mathrm{X}_{2}\right)\right)^{1 / 2}\right]
$$

lies in one sheet of $\Re\left(\mathrm{X}_{2}\right)$ and

$$
\zeta_{1,2}=\left[\zeta_{1},-\left(P\left(\zeta_{1}, \mathrm{X}_{2}\right)\right)^{1 / 2}\right]
$$

lies on another sheet of $\Re\left(\mathrm{X}_{2}\right)$. (See Fig. $4 \mathrm{a}-4 \mathrm{c}$.)

According to the considerations of $\$ 5$

$$
\int_{\mathbb{R}^{*}} S\left(\mathrm{X}_{2}, \zeta\right) f(\zeta) d \zeta, S(\mathrm{X}, \zeta)=\frac{\prod_{k=1}^{3}\left(\sum_{\mu=0}^{N} A_{2 k, \mu} \zeta^{\mu}\right)}{\left[b_{0}(\mathrm{X}) \prod_{k=0}^{6 N-1}\left(\zeta-e_{\kappa}(\mathrm{X})\right)\right]^{1 / 2}}
$$

represents the analytic continuation of $h_{1}(\mathrm{X})$ into the point $\mathrm{X}_{2}$, while $h_{2}\left(\mathrm{X}_{2}\right)$ equals

Therefore

$$
\int_{\varepsilon} S\left(\mathrm{X}_{2}, \zeta\right) f(\zeta) d \zeta
$$

$$
h_{1}\left(\mathrm{X}_{2}\right)-h_{2}\left(\mathrm{X}_{2}\right)=\int_{\Omega-\mathcal{\ell}^{*}} S\left(\mathrm{X}_{2}, \zeta\right) f(\zeta) d \zeta .
$$

Since $\mathrm{X}_{2}$ can be chosen arbitrarily near to $\mathrm{X}_{0}$ and the functions $h_{1}(\mathrm{X})$ and $h_{2}(\mathrm{X})$ are regular at $\mathrm{X}=\mathrm{X}_{0},(2.38)$ yields the theorem.

If through the point $\mathrm{X}_{0}$ several circles $\mathfrak{P}\left(\zeta^{(v)}\right), \zeta^{(v)} \in \mathbb{R}$, pass, then similarly as in $\S 5$ we subdivide $\mathfrak{R}$ into a finite number of parts, $\mathbb{R}=\sum_{\nu=1}^{p} \mathfrak{R}$, such that on each $\Omega_{\nu}$ there lies only one point $\zeta^{(v)}$. Since the saltus of $h(\mathrm{X})$ equals the sum of the jumps of $\int_{\mathfrak{g}} S(\mathrm{X}, \zeta) f(\zeta) d \zeta$, Theorem 2.3 follows in this case as well.

7. Sufficient conditions for analytic continuation of the function $h(\mathrm{X})$ represented in $\mathfrak{u}\left(\mathrm{X}_{0}\right)$ by the integral (2.16). $\S \S 3-6$ were devoted to the study of the behavior of the integral (2.16). The second problem which arises is to study the analytic continuation of the functions represented in a connected part of $a$ by the integral (2.16) outside the domain of representation by this integral. The next chapter will be devoted to the study of this question for a large subclass of functions of the class $\mathfrak{F}(\mathrm{E})$ namely those for which the associate $f$ is a rational function. As we shall see the theory of these functions is in a certain manner a "translated" theory of hyperelliptic integrals. It will 
be, however, of some interest to develop the considerations of $\$ 5$ in order to give here sufficient conditions such that $h(\mathrm{X})$ may be continued along a line.

Definition 2.3. The point $\mathrm{X}_{1}$ will be denoted as an ordinary point of $h(\mathrm{X})$ with respect to its image $e_{\sigma}\left(\mathrm{X}_{1}\right)$ if there exists a neighbourhood $\mathfrak{u}\left(\mathrm{X}_{1}\right)$ of $\mathrm{X}_{1}$, such that to every point $\mathrm{X} \in \mathfrak{U}\left(\mathrm{X}_{1}\right)$ there corresponds one and only one circle $\mathfrak{B}(\zeta)$ of radius $\rho, \rho \geqq \rho_{0}>0$, and $\zeta$ belonging to the neighbourhood $\mathfrak{n}\left[e_{\sigma}\left(\mathrm{X}_{1}\right)\right]$ of $e_{\sigma}\left(\mathrm{X}_{1}\right)$.

Remark 2.7. In this case, we have a one-to-one correspondence, $S(\zeta) \rightarrow \zeta$, between the family $\left[\sum S(\zeta), \zeta \in n\left(e_{\sigma}\left(\mathrm{X}_{1}\right)\right)\right]$ of segments $S(\zeta)=\left[\mathfrak{u}\left(\mathrm{X}_{1}\right)\right] \cap[\mathfrak{P}(\zeta)]$ and the points of the neighbourhood $\mathfrak{n}\left[e_{\sigma}\left(\mathrm{X}_{1}\right)\right]$.

ThEOREM 2.4. Let $\mathrm{g}+\mathrm{g}$ be a curve in $\mathfrak{r}-8$ of finite length, which consists of points $\mathrm{X}_{1}$, each of which is an ordinary point of $h_{1}(\mathrm{X})$ with respect to its image $e_{x}(\mathrm{X})$. Further let us assume that the curve $\zeta=e_{\kappa}(\mathrm{X}), \mathrm{X} \in \mathrm{g}+\mathrm{g}$, lies in the domain of regularity of $f(\zeta)$.

The function $h_{1}(\mathrm{X}), \mathrm{X} \in \mathfrak{U}_{1}\left(\mathrm{X}_{1}\right), \mathrm{X}_{1} \in \mathrm{g}$, given by (2.16) can be analytically continued throughout g.

Proof. The idea of the proof is to show that by starting with the point $\mathrm{X}$ we may repeatedly apply the procedure described in the proof of Theorem 2.2.

The surface $\Im_{\nu}^{*}=\left[\sum \mathfrak{P}(\zeta), \zeta \in \mathbb{R}_{\nu}^{*}\right]$ cuts $\mathfrak{g}$ for the first time at some point, say at $X=X_{2}$, and therefore the segment of $g$ bounded by $X_{1}$ and $X_{2}$ will lie in the regularity domain of $h_{1}^{(\nu)}(\mathrm{X})$.

Repeating our procedure we obtain $h_{1}{ }^{(v)}(\mathrm{X})$ continued along the segment $\mathrm{X}_{2} \mathrm{X}_{3}$ of $\mathfrak{g}$, and so on. In order to prove that after finitely many steps the whole curve $\mathfrak{g}$ will be exhausted it will be sufficient to show that the distance between any two consecutive points $X_{\mathbf{s}}$ and $X_{t+1}$ can be taken larger than a fixed constant which depends on $h_{1}(\mathrm{X})$ and $\mathrm{g}$ but is independent of the location of $\mathrm{X}$, on $\mathrm{g}$.

This fact will easily follow from the following two lemmas.

Lemma 2.1. Let $\mathrm{g}+\mathrm{g}$ be a curve described in Theorem 2.4. To every $\tau$, there exists a continuous $\eta(\tau)$, where

$$
\lim _{\tau \rightarrow 0} \eta(\tau)=0, \eta(\tau)>0 \text { for } \epsilon>0,
$$

such that

$$
\left|e_{\kappa}\left(\mathrm{X}_{1}\right)-e_{\kappa}\left(\mathrm{X}_{2}\right)\right|<\tau
$$

if $\mathrm{X}_{1}$ and $\mathrm{X}_{2}$ are two points of $\mathrm{g}$ for which $\left|\mathrm{X}_{1}-\mathrm{X}_{2}\right|<\eta(\tau)$.

Proof. The existence of the above function follows from the fact that the zero point $e_{k}(\mathrm{X})$ of $P(\zeta, \mathrm{X})=0$, see (2.3), is a continuous function of the coefficients of $P(\zeta, \mathrm{X})$, and that $\left(x_{1}, x_{2}, x_{3}\right)$ vary over a bounded closed set.

In (2.28) we introduced the quantity $\delta=\delta\left(\mathrm{X}_{1}\right)$; now let $\nu$ be the minimum of the $\delta\left(\mathrm{X}_{1}\right)$ when $\mathrm{X}_{1}$ varies over $\mathfrak{p}+\mathrm{p}$, that is, let 


$$
\nu=\min \left[\left|e_{k}(\mathrm{X})-e_{\sigma}(\mathrm{X})\right|, \mathrm{X} \in \mathfrak{g}+\mathrm{g}, \kappa \neq \sigma\right] .
$$

LEMma 2.2. Let $\mathrm{X}_{1}, \mathrm{X}_{2}$ be two points of $\mathrm{g}$ such that

$$
\left|\mathrm{X}_{1}-\mathrm{X}_{2}\right| \leqq \eta(\nu / 2) \text {. }
$$

Then

$$
\left|e_{\kappa}\left(\mathrm{X}_{1}\right)-e_{\sigma}\left(\mathrm{X}_{2}\right)\right| \geqq \nu / 2
$$

Proof. From

$$
\left|e_{\kappa}\left(\mathrm{X}_{1}\right)-e_{\sigma}\left(\mathrm{X}_{2}\right)\right| \geqq\left|e_{\kappa}\left(\mathrm{X}_{1}\right)-e_{\sigma}\left(\mathrm{X}_{1}\right)\right|-\left|e_{\sigma}\left(\mathrm{X}_{2}\right)-e_{\sigma}\left(\mathrm{X}_{1}\right)\right|
$$

(2.43) follows, since from (2.35) we have $\left|e_{\kappa}\left(\mathrm{X}_{1}\right)-e_{\sigma}\left(\mathrm{X}_{1}\right)\right| \geqq \nu$ and from (2.42) and Lemma 2.1 we obtain $\left|e_{\sigma}\left(\mathrm{X}_{2}\right)-e_{\sigma}\left(\mathrm{X}_{1}\right)\right| \leqq \nu / 2$.

If, now, we choose $\epsilon$ (used in the proof of Theorem 2.2) such that

$$
0<\epsilon<\nu / 2 \text {, }
$$

then we obtain for the lower bound $\rho$ of $\left|\mathrm{X}_{\mathrm{s}}-\mathrm{X}_{\mathrm{s}+1}\right|$,

$$
\rho=\eta(\epsilon / 2) \text {. }
$$

Indeed, if $\zeta_{s}^{(v)}=e_{\alpha}\left(\mathrm{X}_{s}\right)$, and $\zeta_{s+1}=e_{\sigma}\left(\mathrm{X}_{s+1}\right)$ is the first following intersection of $g$ with $\mathcal{S}^{*}$ then $\left|\mathrm{X}_{\varepsilon+1}-\mathrm{X}_{\imath}\right| \geqq \eta(\epsilon / 2)$. Were $\left|\mathrm{X}_{s+1}-\mathrm{X}_{s}\right|<\eta(\epsilon / 2)$ and $\sigma=\kappa$, then by Lemma 2.1 we should have $\left|e_{k}\left(\mathrm{X}_{s+1}\right)-e_{\alpha}\left(\mathrm{X}_{s}\right)\right|<\epsilon / 2$ in contradiction with our construction. Were $\zeta_{s+1}=e_{\sigma}\left(\mathrm{X}_{s+1}\right), \sigma \neq \kappa$, and were $\left|\mathrm{X}_{s+1}-\mathrm{X}_{\varepsilon}\right|$ $<\eta(\epsilon / 2)<\eta(\epsilon)$, then by Lemma 2.2 we should have $\left|e_{\sigma}\left(\mathrm{X}_{\varepsilon+1}\right)-e_{x}\left(\mathrm{X}_{\varepsilon}\right)\right|>\epsilon$, which is again in contradiction with our construction.

Remark 2.8. If $\mathfrak{R}$ is sufficiently smooth, if $\Im=E\left[\sum \Im(\zeta), \zeta \in \mathbb{R}\right]$, $\Im(\zeta)=[\mathfrak{P}(\zeta)] \cap \mathfrak{u}\left(\mathrm{X}_{1}\right)$, is sufficiently small, and if $\mathrm{X}_{1} \in \Im$ is an ordinary point of $h(\mathrm{X})$ with respect to its image $e_{\kappa}\left(\mathrm{X}_{1}\right) \in \mathfrak{R}$, then $\mathrm{X}_{1}$ is a regular point of $\Im$.

8. Sufficient conditions in order that a point $X$ be a regular point of a discontinuity surface $\Im$. The notion of a regular point of a discontinuity surface was introduced in $\$ 4$. Here we shall give sufficient conditions in order that a point $\mathrm{X}=\mathrm{X}_{0}$ be a regular point of $\Im$.

THEOREM 2.5. Let $\mathrm{X}_{0}$ be a point of the surface $\Im=\left[\sum \mathfrak{P}(\zeta), \zeta \in \mathfrak{R}\right], \zeta_{0} \in \mathfrak{R}$, through which point passes one and only one circle

$$
\mathfrak{B}\left(\zeta_{0}\right)=\mathrm{E}\left[\sum_{k=1}^{3}\left(y_{k}-w_{k}^{2}\right)=0, \sum_{k=1}^{3} y_{k} w_{k}=0\right]
$$

where $y_{k}=x_{k}-v_{k}, v_{k} \equiv v_{k}\left(\zeta_{0}\right), w_{k} \equiv w_{k}\left(\zeta_{0}\right)$ [see (2.17), (2.18), (2.19)].

$A$ sufficient condition in order that $\mathrm{X}_{0}$ be a regular point is that $\left({ }^{17}\right)$

$$
\sum_{k=1}^{3} b_{k}\left(\xi_{0}, \eta_{0}\right) \frac{\partial\left[v_{k}(\xi, \eta), w_{k}(\xi, \eta)\right]}{\partial[\xi, \eta]_{\xi=\xi_{0}, \eta=\eta_{0}}} \neq 0 .
$$

(17) In the following we use the same symbols $u_{k}, w_{k}$ without distinguishing whether the argument is $\zeta$ or $(\xi, \eta)$. 
Here $b_{k}(\xi, \eta)=w_{k}^{2}(\xi, \eta) \rho^{-2}, k=1,2, b_{3}(\xi, \eta)=1$,

$$
\rho^{2}=w_{1}^{2}(\xi, \eta)+w_{2}^{2}(\xi, \eta), \quad \zeta=\xi+i \eta, \quad \zeta_{0}=\xi_{0}+i \eta_{0} .
$$

Proof. Instead of $x_{1}, x_{2}, x_{3}$ we introduce now a new coordinate system $s_{1}, s_{2}, s_{3}$ such that the origin coincides with the point $\mathrm{X}_{0}$, the tangent to $\mathfrak{P}\left(\zeta_{0}\right)$ at $\mathrm{X}_{0}$ with the positive $s_{1}$ axis $\left({ }^{18}\right)$.

As a formal computation (which is omitted) shows we have

$$
\begin{aligned}
& \left\{\begin{array}{l}
x_{1}=-w_{2} \rho^{-1} s_{1}+w_{1} r^{-1} s_{2}-w_{1} w_{2} r^{-1} \rho^{-1} s_{3}+v_{1}-w_{1} w_{3} \rho^{-1}, \\
x_{2}=w_{1} \rho^{-1} s_{1}+w_{2} r^{-1} s_{2}-w_{1} w_{2} r^{-1} \rho^{-1} s_{3}+v_{2}-w_{1} w_{2} \rho^{-1}, \\
x_{3}=w_{3} r^{-1} s_{2}+\rho r^{-1} s_{3}+v_{3}+\rho,
\end{array}\right. \\
& r=\left(w_{1}^{2}+w_{2}^{2}+w_{3}^{2}\right)^{1 / 2}, \quad \rho=\left(w_{1}^{2}+w_{2}^{2}\right)^{1 / 2} .
\end{aligned}
$$

If in

$$
\sum_{k=1}^{3}\left\{\left[x_{k}-v_{k}(\zeta)\right]^{2}-w_{k}^{2}(\zeta)\right\}=0, \quad \sum_{k=1}^{3}\left[x_{k}-v_{k}(\zeta)\right] w_{k}(\zeta)=0,
$$

we replace the $x_{k}$ by the $s_{k}$, substitute $s_{1}=0$, and express $s_{2}$ and $s_{3}$ as functions of $\xi$ and $\eta$, then the relations

$$
s_{2}=s_{2}\left(\xi, \eta ; \xi_{0}, \eta_{0}\right), \quad s_{3}=s_{3}\left(\xi, \eta, \xi_{0}, \eta_{0}\right)
$$

define a mapping of the $\xi \eta$ plane into the plane $\mathfrak{A}\left(\zeta_{0}\right)$, see (2.19), in which the circle $\mathfrak{P}\left(\zeta_{0}\right)$ lies. If the Jacobian of transformation (2.48) does not vanish at $(\xi, \eta)=\left(\xi_{0}, \eta_{0}\right)$ it means that the mapping of the plane into the plane $\mathscr{A}\left(\zeta_{0}\right)$ is one-to-one at the point $\left(\xi_{0}, \eta_{0}\right)$.

Since in a sufficiently small neighborhood $\mathfrak{u}\left(\mathrm{X}_{0}\right)$ of $\mathrm{X}_{0}$, the arcs

$$
[\mathfrak{P}(\zeta)] \cap\left[\mathfrak{u}\left(\mathrm{X}_{0}\right)\right]
$$

of the circles $\mathfrak{P}(\zeta), \zeta \in \mathfrak{n}\left(\zeta_{0}\right)$, are perpendicular to $\mathfrak{A}\left(\zeta_{0}\right)$, the correspondence between the points $\zeta, \zeta \in \mathfrak{n}\left(\zeta_{0}\right)$, and arcs (2.49) is one-to-one.

A formal computation shows that the Jacobian of the transformation (2.48) at $\left(\xi_{0}, \eta_{0}\right)$ equals the left-hand side of $(2.45)$, which yields the statement of Theorem 2.5 .

\section{THE BeHAVIOR IN THE LARGE OF THE FUNCTIONS OF THE CLASS TC(E) WITH A RATIONAL ASSOCIATE}

1. Introduction. As we showed in Chapter II, the integral (2.16) represents a two-valued function which is defined at every point of the domain a of the three-dimensional euclidean $x_{1}, x_{2}, x_{3}$ space.

In every connected region, $\mathfrak{b}$, of the $x_{1}, x_{2}, x_{3}$ space $\left({ }^{19}\right)$ the function obtained

(18) The direction of the axes $s_{2}$ and $s_{3}$ are not yet uniquely determined since we may rotate the $s_{2} s_{3}$ plane around the $s_{1}$ axis, but for our purposes it is unessential how we choose the $s_{2}$ and $s_{3}$ axis in the plane which is normal to the $\mathfrak{B}\left(\zeta_{0}\right)$ at $\mathrm{X}_{0}$.

(19) $\mathrm{b}$ is not necessarily schlicht in the euclidean $x_{1}, x_{2}, x_{8}$ space; it may be situated in two space sheets, that is, a three-dimensional analogue of a plane domain which lies on two sheets of a Riemann surface. 
represents a regular harmonic function. In general, this,function can be analytically continued outside the domain $\mathfrak{b}$, where it is represented by (2.16).

In the present chapter we shall study these functions in the large, that is, in the whole domain of regularity of the function under consideration. As was indicated in chapter II, $\$ \S 5$ and 7 ; the main tool for the analytic continuation of our functions outside $\mathfrak{b}$ consists of a suitable change of integration in the $\zeta$ plane.

On the other hand if we assume that $f(\zeta)$ in $(2.16)$ is a rational function, (2.16) becomes a hyperelliptic integral. The theory of these functions has been developed to a great extent, in particular the question of how these functions change when the integration path (with fixed initial and end point) is varied has been investigated in detail. As was stressed in chapter I our investigation reduces itself to the "translation" of classical results of the theory of hyperelliptic integrals $\left({ }^{20}\right)$. Naturally, the fact that the Riemann surface on which our integrals are defined depends upon the variables $x_{1}, x_{2}, x_{3}$ causes additional complications.

2. Reduction to the Weierstrass normal form. In order to apply the results of theory of hyperelliptic integrals, in particular to use the techniques developed by Weierstrass, we have to reduce our integrals to the normal form employed by Weierstrass, see [7].

First of all we introduce a new variable of integration( $\left.{ }^{21}\right)$,

$$
\xi=\left[\zeta-e_{0}(\mathrm{X})\right]^{-1}
$$

(that is, $\zeta=\xi^{-1}+e_{0}(\mathrm{X})$ ). The Riemann surface over the $\xi$-plane which we obtain from $\Re(\mathrm{X})$ by applying the transformation (3.1) will be denoted by $\mathfrak{W}(\mathrm{X}), \mathrm{X} \in \mathfrak{r}-\mathbb{B} . \mathfrak{B}(\mathrm{X})$ has $(6 N-1)$ branch points

$$
a_{k}(\mathrm{X})=\left[e_{\kappa+1}(\mathrm{X})-e_{0}(\mathrm{X})\right]^{-1}, \quad \kappa=0,1, \cdots, 6 N-2,
$$

and a branch point at $\xi=\infty$.

Let

$$
\eta= \pm[R(\xi, \mathrm{X})]^{1 / 2}
$$

where

$$
R(\xi, \mathrm{X})=\prod_{k=0}^{6 N-2}\left[\xi-a_{k}(\mathrm{X})\right]
$$

(30) The limitation of space makes it impossible for us to discuss all results of the theory of hyperelliptic integrals from this point of view. We shall limit ourselves to discussion of one typical case, but we should like to emphasize that the considerations of various other theorems of the theory of hyperelliptic integrals lead to results which are of interest for the theory of harmonic functions in three variables, and of importance in the applications of this theory.

(21) We notice that by writing as argument a complex number, $\xi$, we do not completely describe a point of the Riemann surface $\mathfrak{W}(\mathrm{X})$, since it is necessary in addition to the location of the point in the (schlicht) plane to indicate the sheet in which the point is situated. (Many authors use for point the symbol $(\xi, \eta)$ ). However in order to avoid complicated formulae, in the following we shall use only a single letter $\xi$. 
We introduce the expressions

and $\left({ }^{22}\right)$

$$
S(\xi, \mathrm{X})=\mathrm{E}(\mathrm{X}, \zeta) \eta \frac{d \zeta}{d \xi}=\frac{\xi^{3 N-2} \prod_{k=1}^{3}\left[\sum_{\mu=1}^{N} A_{2 k, \mu}\left(\xi^{-1}+e_{0}(\mathrm{X})\right)^{\mu}\right]}{\left\{b_{0}(\mathrm{X}) \prod_{k=0}^{6 N-2}\left[e_{\kappa+1}(\mathrm{X})-e_{0}(\mathrm{X})\right]\right\}^{1 / 2}}
$$

$$
g(\xi, \mathrm{X})=f\left[\xi^{-1}-e_{0}(\mathrm{X})\right]=\frac{\sum_{n=0}^{M_{1}} B_{1, n}\left(\xi^{-1}-e_{0}(\mathrm{X})\right)^{n}}{\sum_{n=0}^{M_{2}} B_{2, n}\left(\xi^{-1}-e_{0}(\mathrm{X})\right)^{n}} .
$$

The operator (2.11) now assumes the form

$$
\int_{\xi_{0}(\mathrm{X})}^{\xi_{1}(\mathbf{X})} \frac{S(\xi, \mathrm{X}) g(\xi, \mathrm{X}) d \xi}{\eta}, \quad \mathrm{X} \in \mathfrak{u}\left(\mathrm{X}_{0}\right) .
$$

Here $\xi_{0}(\mathrm{X})$ and $\xi_{1}(\mathrm{X})$ are the initial and the end point of the image of $\mathfrak{I}$ on the Riemann surface $\mathfrak{W}(\mathrm{X})$. (That is to say,

$$
\begin{aligned}
& \xi_{0}(\mathrm{X})=\left[\zeta_{0}-e_{0}(\mathrm{X})\right]^{-1}, \\
& \xi_{1}(\mathrm{X})=\left[\zeta_{1}-e_{0}(\mathrm{X})\right]^{-1} \text {.) }
\end{aligned}
$$

3. The domain $\mathfrak{p}$. In using the classical approach to the study of (3.5) we have to take into account that the integrand depends in addition to the integration variable $\xi$, upon $x_{1}, x_{2}, x_{3}$, which can be considered as parametric variables.

Following classical considerations, in particular the methods due to Weierstrass (see [7]), we introduce on the Riemann surface $\mathfrak{W}(\mathrm{X})$ integrals of the first, second and third kinds, and determine their periods.

$\mathfrak{B}(\mathrm{X})$ is a multiply connected domain, and in order to determine the periods we must, by conveniently chosen loop cuts $\left({ }^{23}\right) \mathbb{E}_{2 k}(X)$ and crosscuts $\mathbb{E}_{2 k+1}(\mathrm{X}), \kappa=0,1, \cdots, \rho-1, \rho=3 N-1$, transform $\mathfrak{W}(\mathrm{X})$ into a simply connected domain, $\mathfrak{B}^{*}(\mathrm{X}) . \mathfrak{W}(\mathrm{X})$ depends upon $\mathrm{X}$, since its branch points $a_{k}(\mathrm{X})$ vary with $\mathrm{X}$. In order to determine in a unique way the periods [which can be defined as integrals over the cuts $\left.\mathcal{E}_{\kappa}(\mathrm{X})\right]$, it is necessary to make certain restrictions upon the domain in which $\mathrm{X}$ varies.

$\mathfrak{r}-\mathfrak{s}$ is a connected three-dimensional domain. In general, not every closed simple curve in $\mathfrak{r}-8$ can be reduced to a point without cutting 8 . On the other hand, it is possible by cutting it by a segment of an appropriately chosen surface, $\mathfrak{T}$, to obtain a domain $\mathfrak{p}=\mathfrak{r}-\mathfrak{b}-\mathfrak{T}$ which possesses the property that every

(22) Since now $f$ is assumed to be a rational function of $\zeta$ it can be written as quotient of two polynomials.

(23) As usual all loopcuts lie in one sheet, the crosscuts lie partially in one, partially in another sheet. Each $\xi_{\nu}\left(\mathrm{X}_{0}\right)$ intersects $\xi_{\nu-1}\left(\mathrm{X}_{0}\right)$ and $\xi_{\nu+1}\left(\mathrm{X}_{0}\right)$ exactly at one point each. 
closed curve in $\mathfrak{p}$ can be reduced to a point in $\mathfrak{p}$. In addition we may require that every boundary point of $\mathfrak{T}$ which is not at infinity belongs to 8 .

Let $\mathrm{X}_{0}$ be some fixed point of $\mathfrak{p}$. We rearrange the $a_{k}\left(\mathrm{X}_{0}\right)$ in such a manner that

$$
a_{0}\left(\mathrm{X}_{0}\right)<a_{1}\left(\mathrm{X}_{0}\right)<\cdots<a_{2 \rho}\left(\mathrm{X}_{0}\right), \quad \rho=3 N-1 \text {. }
$$

( $A<B$ means that $\operatorname{Re} A \leqq \operatorname{Re} B$, and $\operatorname{Im} A<\operatorname{Im} B$ if $\operatorname{Re} \mathrm{A}=\operatorname{Re} B$.)

We connect both sheets of $\mathfrak{B}\left(\mathrm{X}_{0}\right)$ along the segments $\left[a_{2 \kappa}\left(\mathrm{X}_{0}\right), a_{2 \kappa+1}(\mathrm{X})\right]$ of straight lines, $\kappa=0,1, \cdots, a_{2 \rho+1}\left(\mathrm{X}_{0}\right)=\infty$. Each of the (above-mentioned) cuts $\mathbb{E}_{k}\left(\mathrm{X}_{0}\right)$ is supposed to consist of two segments of straight lines which are parallel to $\left[a_{\kappa}\left(\mathrm{X}_{0}\right), a_{\kappa+1}\left(\mathrm{X}_{0}\right)\right]$ and of two halfcircles with the centers at $a_{k}\left(\mathrm{X}_{0}\right)$ and $a_{\kappa+1}\left(\mathrm{X}_{0}\right)$, respectively. If now $\mathrm{X}$ varies in $\mathfrak{p}$ then the $a_{k}(\mathrm{X})$, being functions of $X$, move, and therefore the $\mho_{k}(X)$ move also. Since however the points $\mathrm{X}$ for which two $a_{k}(\mathrm{X})$ coincide are excluded it is possible to arrange the $\mathbb{E}_{k}(X)$ 's so that they change continuously $\left({ }^{24}\right)$. Since further any closed curve in $\mathfrak{p}$ can be reduced to a point, the sense of direction on every $\xi_{k}(\mathrm{X})$ is determined in a unique manner.

4. Period functions. In order to define the normal integrals we introduce the Weierstrass $H$ functions, defined on $\mathfrak{W}(\mathrm{X})$.

We write

$$
N(\xi, \mathrm{X})=\prod_{n=1}^{p}\left[\xi-a_{2 n-1}(\mathrm{X})\right], \quad Q(\xi, \mathrm{X})=\prod_{n=0}^{p}\left[\xi-a_{2 n}(\mathrm{X})\right]
$$

and following Weierstrass (see [7, pp. 340-343]) define $\left({ }^{25}\right)$

$$
\left.\begin{array}{rl}
H(\xi, \mathrm{X})_{\alpha} & =N(\xi, \mathrm{X}) / 2 \eta\left[\xi-a_{2 \alpha-1}(\mathrm{X})\right] \\
H^{*}(\xi, \mathrm{X})_{\alpha} & =-\frac{Q\left[a_{2 \alpha-1}(\mathrm{X}), \mathrm{X}\right] \cdot N(\xi, \mathrm{X})}{N^{\prime}\left[a_{2 \alpha-1}(\mathrm{X}), \mathrm{X}\right] \cdot 2 \eta\left[\xi-a_{2 \alpha-1}(\mathrm{X})\right]^{2}},
\end{array}\right\} \alpha=1,2, \cdots, \rho,
$$

The "period functions," that is, the periods of the normal integrals of the first and second kind, are defined by $\left({ }^{26}\right)$

(24) We drop the requirement that the $\left[a_{k}(\mathrm{X}), a_{k+1}(\mathrm{X})\right]$ are segments of straight lines and consequently each cut will consist of two lines of a form similar to the corresponding $\left[a_{k}(\mathrm{X}), a_{k+1}(\mathrm{X})\right]$ and two halfcircles with the centers at $a_{k}(\mathrm{X})$ and $a_{k+1}(\mathrm{X})$, respectively. Since only the topological structure of the cuts is of importance for our purposes, the form of the cuts is unessential.

(25) We use here Weierstress' original notation.

(26) Note that in contrast to $\mathbb{R}$ which can be situated either in one or in another sheet, $\mathfrak{E}_{\nu}(\mathrm{X})$ are defined directly on the Riemann surface $\mathfrak{W}(\mathrm{X})$. 


$$
\begin{array}{ll}
2 \omega_{\alpha \beta}(\mathrm{X})=2 \omega_{\alpha}^{(2 \beta-1)}(\mathrm{X}), & 2 \eta_{\alpha \beta}(\mathrm{X})=2 \eta_{\alpha}^{(2 \beta-1)}(\mathrm{X}), \\
2 \omega_{\alpha \beta}^{*}(\mathrm{X})=2 \sum_{\delta=0}^{\beta-1} \omega_{\alpha}^{2 \delta}(\mathrm{X}), & 2 \eta_{\alpha \beta}^{*}(\mathrm{X})=2 \sum_{\delta=0}^{\beta-1} \eta_{\alpha}^{2 \delta}(\mathrm{X}),
\end{array}
$$

where

$$
\begin{aligned}
2 \omega_{\beta}^{\alpha}(\mathrm{X})= & \int_{\mathfrak{E}_{2 \beta}(\mathrm{X})} H(\xi, \mathrm{X})_{\alpha} d \xi=\int_{\mathfrak{E}_{2 \beta}(\mathrm{X})} \frac{N(\xi, \mathrm{X})}{\xi-a_{2 \alpha-1}(\mathrm{X})} \frac{d \xi}{\eta}, \\
2 \eta_{\beta}^{\alpha}(\mathrm{X})=\int_{\mathfrak{E}_{2 \beta}(\mathrm{X})} H^{*}(\xi, \mathrm{X})_{\beta} d \xi . & \alpha=1,2, \cdots, \rho ; \beta=1,2, \cdots, \rho,
\end{aligned}
$$

Remark 3.2. We note that $\omega_{\beta}^{\alpha}(\mathrm{X})$ are entire functions of the $a_{\kappa}(\mathrm{X})$ (see $[7$, p. 359]) and that

$$
2 \eta_{\beta}^{\alpha}(\mathrm{X})=-2 \frac{Q\left[a_{2 \beta-1}(\mathrm{X}), \mathrm{X}\right]}{N^{\prime}\left[a_{2 \beta-1}(\mathrm{X}), \mathrm{X}\right]} \cdot \frac{\partial \omega_{\beta}^{\alpha}\left(\mathrm{X}_{1}\right)}{\partial a_{2 \beta-1}(\mathrm{X})} .
$$

The functions $\omega_{\alpha \beta}(\mathrm{X}), \omega_{\alpha \beta}^{*}(\mathrm{X}), \eta_{\alpha \beta}(\mathrm{X})$ and $\eta_{\alpha \beta}^{*}(\mathrm{X})$ are single-valued in $\mathfrak{p}$. If, however, starting from a point $\mathrm{X}_{0}$ and moving along a closed line (which intersects $\mathfrak{T}$ and which can not be reduced to a point without intersecting $\mathbb{8}$ ) we return to the point $\mathrm{X}_{0}$, then, in general, the integration curve will not necessarily coincide with the starting curve and each $\omega_{\alpha \beta}(\mathrm{X})$ will assume a new value. Since however $\int H\left(\xi, \mathrm{X}_{0}\right)_{\alpha} d \xi$ (where the integration is taken over any closed curve) equals

$$
\sum_{\beta=1}^{\rho}\left[2 n_{\beta} \omega_{\alpha \beta}\left(\mathrm{X}_{0}\right)+2 n_{\beta}^{*} \omega_{\alpha \beta}^{*}\left(\mathrm{X}_{0}\right)\right]
$$

$n_{\beta}, n_{\beta}{ }^{*}$ being integers, we obtain the following:

TheOREM 3.1. The functions $\omega_{\alpha \beta}(\mathrm{X}), \omega_{\alpha \beta}^{*}(\mathrm{X}), \eta_{\alpha \beta}(\mathrm{X})$ and $\eta_{\alpha \beta}^{*}(\mathrm{X})$ are, in general, infinitely many-valued functions which are defined in $\mathfrak{r}-8$.

If we move along a closed curve in $\mathfrak{r}-8$ which can not be reduced to a point by a one-to-one continuous transformation, then $\omega_{\alpha \beta}(\mathrm{X})$ and $\omega_{\alpha \beta}^{*}(\mathrm{X})$ increase by an expression of the form (3.12). Similar results hold for the $\eta_{\alpha \beta}(\mathrm{X})$ and $\eta_{\alpha \beta}^{*}(\mathrm{X})$.

We note further that from a classical theorem of Fuchs [5] on periods of normal integrals the following result obtains: The functions $\omega_{\alpha \beta}(\mathrm{X}), \cdots$ considered as functions of $x_{1}$, satisfy an ordinary differential equation whose coefficients are algebraic functions of $x_{1}, x_{2}, x_{3}$. Similar equations hold when $\omega_{\alpha \beta}(\mathrm{X}), \ldots$ are considered as functions of $x_{2}$ as well as of $x_{3}$.

5. The normal integrals of the first, second and third kinds. Following Weierstrass we now define in $p$ the normal integrals of the first, second and third kind, namely 


$$
\begin{aligned}
& \left.\begin{array}{rl}
J(\xi, \mathrm{X})_{\alpha} & =\int_{a_{0}(\mathrm{X})}^{\xi(\mathrm{X})} H\left(\xi^{\prime}, \mathrm{X}\right)_{\alpha} d \xi^{\prime}, \\
J^{*}(\xi, \mathrm{X})_{\alpha} & =\int_{a_{0}(\mathrm{X})}^{\xi(\mathrm{X})} H^{*}\left(\xi^{\prime}, \mathrm{X}\right)_{\alpha} d \xi^{\prime},
\end{array}\right\} \\
& J\left(\xi, \xi^{(1)}, \xi^{(0)} ; \mathrm{X}\right)=\int_{a_{0}(\mathrm{X})}^{\xi(\mathrm{X})}\left[H\left(\xi^{(1)}(\mathrm{X}), \xi^{\prime} ; \mathrm{X}\right)-H\left(\xi^{(0)}(\mathrm{X}), \xi^{\prime} ; \mathrm{X}\right)\right] d \xi^{\prime}, \\
& \xi \equiv \xi(\mathrm{X})=\left[\zeta-e_{0}(\mathrm{X})\right]^{-1}, \quad \xi^{(1)} \equiv \xi^{(1)}(\mathrm{X})=\left[\zeta^{(1)}-e_{0}(\mathrm{X})\right]^{-1}, \\
& \xi^{(0)} \equiv \xi^{(0)}(\mathrm{X})=\left[\zeta^{(0)}-e_{0}(\mathrm{X})\right] \\
& \alpha=1,2, \cdots, \rho,
\end{aligned}
$$

According to our previous considerations the functions (3.13) can have singularities on the circles

$$
\mathfrak{B}\left[e_{0}\right] \text { and } \mathfrak{P}[\zeta]
$$

in addition to those of 8 .

The function (3.14) can be singular also on

$$
\mathfrak{B}\left[\zeta^{(1)}\right] \text { and } \mathfrak{B}\left[\zeta^{(0)}\right] \text {. }
$$

If now we continue analytically the functions (3.13) and (3.14) along a line, which line can not be reduced to a point without cutting 8 , then the functions $J(\xi, \mathrm{X})_{\alpha}$ and $J^{*}(\xi, \mathrm{X})_{\alpha}$ increase by a function. These functions are (3.12) in the case of $J(\xi, \mathrm{X})_{\alpha}$ and analogous expressions with $\omega$ 's replaced by $\eta$ 's in the case of $J^{*}(\xi, \mathrm{X})_{\alpha}$.

In addition $J$ and $J^{*}$ will, in general, increase by a function if we move, in $\mathfrak{p}$, along a closed curve which cannot be reduced to a point without cutting either $\mathfrak{B}\left[e_{0}\right]$ or $\mathfrak{B}[\zeta]$.

Analogous results are obtained for (3.14).

6. The representation of the integrals (2.16) in terms of normal integrals of the first, second and third kind. In this chapter we assume that the function $f$, appearing in (2.16), is a rational function of $\zeta$. Then $S(\xi, \mathrm{X}) g(\xi, \mathrm{X})$ in (3.5) will be also a rational function of $\xi$. In the theory of hyperelliptic integrals it is shown that every such integral can be expressed as a sum of normal integrals with constant coefficients.

This theorem can be immediately generalized to the problem which is being considered. In this case the above-mentioned coefficients are no longer constant but are, as we shall see, algebraic functions of $x_{1}, x_{2}, x_{3}$.

TheOREM 3.2. An integral of the form (2.16) with a rational associate $f$ can be represented as a sum of normal integrals of the first, second and third kind associated with the Riemann surface $\mathfrak{B}(\mathrm{X})$ of the function $[R(\xi, \mathrm{X})]^{1 / 2}$; introduced in (3.2). That is to say, integral (2.16) with a rational associate can be represented in the form 


$$
\begin{aligned}
\sum_{\nu=1}^{r} C_{\nu}(\mathrm{X})\left\{J \left[\xi_{1}(\mathrm{X}) ;\right.\right. & \left.\left.\xi^{(1)}(\mathrm{X}), \xi^{(0)}(\mathrm{X}) ; \mathrm{X}\right]-J\left[\xi_{0}(\mathrm{X}) ; \xi^{(1)}(\mathrm{X}), \xi^{(0)}(\mathrm{X}) ; \mathrm{X}\right]\right\} \\
& -\sum_{\alpha=1}^{p}\left\{g_{\alpha}^{*}(\mathrm{X})\left[J\left(\xi_{1}(\mathrm{X}) ; \mathrm{X}\right)_{\alpha}-J\left(\xi_{0}(\mathrm{X}) ; \mathrm{X}\right)_{\alpha}\right]\right. \\
& \left.+g_{\alpha}(\mathrm{X})\left[J^{*}\left(\xi_{1}(\mathrm{X}) ; \mathrm{X}\right)_{\alpha}-J^{*}\left(\xi_{0}(\mathrm{X}) ; \mathrm{X}\right)_{\alpha}\right]\right\} \\
& +\sum_{\nu=1}^{n}\left[F_{\nu}\left(\xi_{1}(\mathrm{X}) ; \mathrm{X}\right)-F_{\nu}\left(\xi_{0}(\mathrm{X}) ; \mathrm{X}\right)\right]
\end{aligned}
$$

Here $\xi_{\kappa}(\mathrm{X})=\left[\zeta_{\kappa}-e_{0}(\mathrm{X})\right]^{-1}, \kappa=0,1$, and the coefficients $C_{\nu}(\mathrm{X}), g_{\alpha}(\mathrm{X})$ and $g_{\alpha}^{*}(\mathrm{X})$ of the development are algebraic functions of $x_{1}, x_{2} ; x_{3} . F_{\nu}$ are algebraic functions of $x_{1}, x_{2}, x_{3}$.

Proof. According to $[7$, p. 264] every, rational function of $\xi$ and $\eta$, and therefore in particular the integrand of (3.5), can be represented in the form

$$
S(\xi, \mathrm{X}) g(\xi, \mathrm{X}) \equiv F(\xi, \mathrm{X})=\sum_{\nu=1}^{r} C_{\nu}(\mathrm{X}) H\left(\xi_{\nu}, \xi ; \mathrm{X}\right)
$$

$$
\begin{aligned}
& -\sum_{\alpha=1}^{\rho}\left\{g_{\alpha}^{*}(\mathrm{X}) H(\xi, \mathrm{X})_{\alpha}-g_{\alpha}(\mathrm{X}) H^{*}(\xi, \mathrm{X})_{\alpha}\right\} \\
& +\frac{d}{d \xi}\left[\sum_{\nu=1}^{n} F_{\nu}(\xi, \mathrm{X})\right] .
\end{aligned}
$$

We have therefore to show that the coefficients $C_{\nu}(\mathrm{X}), g_{\alpha}^{*}(\mathrm{X}), g_{\alpha}(\mathrm{X})$ are algebraic functions of $x_{1}, x_{2}, x_{3}$, and that $F_{\nu}(\xi, \mathrm{X})$ are algebraic functions of $\xi$ and $x_{1}, x_{2}, x_{3}$. According to $[7$, p. 264]

$$
C_{\nu}(\mathrm{X})=\left[F\left(\xi_{t}^{\prime \prime}, \mathrm{X}\right) \frac{d \xi_{t}^{\prime}}{d t}\right]_{t-1}
$$

that is, the $C_{\nu}(\mathrm{X})$ is equal to the coefficient of the power of $t^{-1}\left({ }^{27}\right)$ of the series development of the local uniformizing variable $t$, at a point at which the integrand becomes infinite. Since $F(\xi, \mathrm{X})$ is an algebraic function of $\xi$ and $x_{1}, x_{2}, x_{3}$, and the values of $\xi$ for which $F$ becomes infinite (that is, the zeros of the denominator of $S(\xi, \mathrm{X}) g(\xi, \mathrm{X})$ ) as well as the coefficients of the development can be differentiations and algebraic operations, the $C_{\nu}(\mathrm{X})$ are algebraic functions of $x_{1}, x_{2}, x_{3}$.

The same holds for the functions

$$
C_{\nu,-n}(\mathrm{X})=-\frac{1}{n}\left[F\left(\xi_{t}^{\prime \prime}\right) \frac{d \xi_{t}^{\prime}}{d t}\right]_{t-n-1}, \quad n>0,
$$

and

${ }^{(27)}$ We denote by $[s]_{t^{k}}$ the coefficient of $t^{k}$ of the development of $s$. 


$$
C^{(\alpha)}(\mathrm{X})=\left[F\left(\xi_{t}^{(\alpha)}\right) \frac{d \xi_{t}^{(\alpha)}}{d t}\right]_{t^{0}}
$$

where $\xi^{(\alpha)}$ are the branch points $a_{1}(\mathrm{X}), a_{3}(\mathrm{X}), \cdots, a_{2 \rho-1}(\mathrm{X})$, see $[7, \mathrm{p} .340]$.

$$
F_{v}(\xi, \mathrm{X})=-\sum_{n} C_{v_{1}-r}(\mathrm{X})\left[H\left(\xi, \xi_{t}^{\prime}, \mathrm{X}\right) \frac{d \xi_{t}^{\prime}}{d t}\right]_{t^{n-1}}
$$

where $H\left(\xi, \xi^{*}, \mathrm{X}\right)$ is the function introduced in (3.9). (They are obviously algebraic functions of $\xi$ and $x_{1}, x_{2}, x_{3}$.) Similarly

$$
\begin{aligned}
& \sum_{n} C_{\nu,-n}(\mathrm{X})\left[H\left(\xi_{t}, \mathrm{X}\right)_{\alpha} \frac{d \xi_{t}^{\prime}}{d t}\right]_{t^{n-1}}=H_{v, \alpha}(\mathrm{X}), \\
& \sum_{n} C_{v,-n}\left[H^{*}\left(\xi_{t}^{\prime}, \mathrm{X}\right)_{\alpha} \frac{d \xi_{t}}{d t}\right]_{t^{n-1}}=H_{v, \alpha}^{*}(\mathrm{X})
\end{aligned}
$$

where $H(\xi, \mathrm{X})_{\alpha}, H^{*}(\xi, \mathrm{X})_{\alpha}$ are the functions introduced in (3.9). $H_{\nu, \alpha}(\mathrm{X})$, $H_{v, \alpha}^{*}(\mathrm{X})$ are algebraic functions of $x_{1}, x_{2}, x_{3}$, and therefore

$$
\begin{aligned}
& g_{\alpha}(\mathrm{X})=\sum_{\nu=1}^{r} H_{\nu, \alpha}(\mathrm{X}), \\
& g_{\alpha}^{*}(\mathrm{X})=\sum_{\nu=1}^{r} H_{\nu, \alpha}^{*}(\mathrm{X})-C^{(\alpha)}(\mathrm{X})
\end{aligned}
$$

are also algebraic functions, which completes our proof.

7. The application of $\theta$ functions. The introduction of $\theta$ functions and representation of the integrals of the second and third kinds using these functions in terms of the normal integral of the first kind is one of the important achievements of the theory of algebraic functions.

The interpretation of these results in the frame of the theory of harmonic functions in three variables yields the following result:

In chapter II the subclass $\delta\left[\mathbf{E}, \zeta_{0}, \zeta_{1}\right]$ was introduced. Since $g(\zeta)$ is an arbitrary rational function of $\zeta$, these subclasses consist of infinitely many functions.

We associate with such a subclass of $\theta$ functions, which functions we obtain in the usual manner, substituting for the periods (which are constants in the classical theory) the period function introduced in $\$ 4$ of the present chapter.

The functions

$$
\theta\left(u_{1} \cdots u_{p} ; \mathrm{X}\right)
$$

depend, in addition to the $\rho$ variable $u_{\rho}, \rho=3 N-1$, upon $x_{1}, x_{2}, x_{3}$.

The subclass $S\left[E, \zeta_{0}, \zeta\right]$ possesses a finite basis with respect to algebrologarithmic functions and the $\theta$ functions described above; that is, there exist 
$\rho$ functions $J(\xi, \mathrm{X})_{\alpha}$ such that every $h(X)$ belonging to $S\left[\mathrm{E}, \zeta_{0}, \zeta_{1}\right]$ can be expressed in closed form, which form involves only algebro-logarithmic functions and the $\theta$ functions and their derivatives, where the arguments $u_{1}, \cdots, u_{p}$ of the $\theta$ functions are replaced by linear combinations of the $J(\xi, \mathrm{X})_{\alpha}$ with coefficients which are algebraic functions of $x_{1}, x_{2}, x_{3}$.

We proceed to more detailed formulation of the result.

We write following Weierstrass [7, pp. 532-533]

$$
\begin{aligned}
\epsilon\left(v_{1}, \cdots, v_{\rho} ; X\right) & =\sum_{\beta=1}^{p} \sum_{\nu=1}^{p} 2 \epsilon_{\beta \gamma}(X) v_{\beta} v_{\gamma} \\
\epsilon_{\beta \gamma}(X) & =\sum_{\alpha=1}^{\rho} \omega_{\alpha \beta}(X) \eta_{\alpha \gamma}(X)
\end{aligned}
$$

where $\omega_{\alpha \beta}(\mathrm{X})$ and $\eta_{\alpha \gamma}(\mathrm{X})$ are period functions introduced in $\S 4$. Further, we write

$$
\chi\left(n_{1}, \cdots, n_{\rho} ; \mathrm{X}\right)=\pi i \sum_{\alpha=1}^{\rho} \sum_{\beta=1}^{\rho} n_{\alpha} n_{\beta}\left[\sum_{\gamma=1}^{\rho} \frac{(\omega(\mathrm{X}))_{\alpha \beta} \omega_{\alpha \beta}^{*}(\mathrm{X})}{\omega(\mathrm{X})}\right]
$$

where $\omega(\mathrm{X})=\left|\omega_{\alpha \beta}(\mathrm{X})\right|$ and $(\omega(\mathrm{X}))_{\alpha \beta}=\left[\partial \omega / \partial \omega_{\alpha \beta}\right]$. Then

$$
\theta\left(u_{1}, \cdots, u_{\rho} ; X\right)=\exp \left[\epsilon\left(v_{1}, \cdots, v_{\rho} ; X\right)\right] \cdot \theta\left(v_{1}, \cdots, v_{p} ; X\right)
$$

where

$$
\theta\left(v_{1}, \cdots, v_{p} ; \mathrm{X}\right)=\sum_{n_{k}=-\infty}^{\infty} \exp \left[\chi\left(n_{1}, \cdots, n_{\rho} ; \mathrm{X}\right)\right]+2 \pi i \sum_{\nu=1}^{p} n_{\nu} v_{\nu}
$$

(see $[7$, p. 531$]$ ) and

$$
v_{\rho}=\sum_{\alpha=1}^{\rho} \frac{[\omega(\mathrm{X})]_{\alpha \beta}}{2 \omega(\mathrm{X})} \boldsymbol{u}_{\alpha} .
$$

Remark. We shall in the following use the abbreviation

$$
\theta^{(\alpha)}\left(u_{1}, \cdots, u_{p} ; X\right)=\frac{\partial \theta\left(u_{1}, \cdots, u_{p} ; X\right)}{\partial u_{\alpha}}
$$

In order to obtain the mentioned representation of the integrals of the second and third kind in terms of the integrals of the first kind, we have to introduce certain new functions of $\mathrm{X}$, namely

$$
w_{\beta}(\mathrm{X})=2^{-1} \sum_{\nu=1}^{2 \rho-2} J\left(p_{\nu}(\mathrm{X})\right)_{\beta}
$$


(see $\left[7\right.$, p. 597]) where the $p_{v}(\mathrm{X})$ have to be determined in the following manner. Consider

$$
\sum_{\beta=1}^{\rho} K_{\beta} G(\xi, \mathrm{X})_{\beta} / \sum_{\beta=1}^{\rho} K_{\beta}^{*} G(\xi, \mathrm{X})
$$

(see $\left[7\right.$, p. 594]) where $K_{\beta}$ and $K_{\beta}^{*}$ are arbitrary constants and $G(\xi, \mathrm{X})_{\alpha}$ $=\left[H(\xi, \mathrm{X})_{\alpha}\right] \cdot f(\xi)_{2}$ (see $[7$, p. 109]). If we determine the points for which the denominator vanishes and the numerator is not zero we obtain the $(2 \rho-2)$ points $p_{\nu}(\mathrm{X})$.

Remark 3.1. The points $p_{\nu}(\mathrm{X})$ depend upon the choice of the constants $K_{\beta}$, $K_{\beta}^{*}$, the right-hand side of $(3.30)$ is however independent of these constants (see $[7$, p. 594]).

According to $[7$, p. 597] we can now express the integrals of second and third kind in terms of integrals of the first kind. We have namely

$$
\begin{aligned}
J^{*}(\xi, \mathrm{X})_{\alpha}-J^{*}\left(\xi_{0}, \mathbf{X}\right)_{\alpha}= & \frac{\theta^{(\alpha)}\left[J(\xi, \mathbf{X})_{1}-w_{1}(\mathrm{X})-J\left(a_{\alpha}, \mathrm{X}\right)_{1}, \cdots, \mathrm{X}\right]}{\theta\left[J(\xi, \mathrm{X})_{1}-w_{1}(\mathrm{X})-J\left(a_{\alpha}, \mathrm{X}\right)_{1}, \cdots, \mathrm{X}\right]} \\
& -\frac{\theta^{(\alpha)}\left[J\left(\xi_{0}, \mathrm{X}\right)_{1}-w_{1}(\mathrm{X})-J\left(a_{\alpha}, \mathrm{X}\right)_{1}, \cdots, \mathrm{X}\right]}{\theta\left[J\left(\xi_{0}, \mathrm{X}\right)_{1}-w_{1}(\mathrm{X})-J\left(a_{\alpha}, \mathrm{X}\right), \cdots, \mathrm{X}\right]}
\end{aligned}
$$

(see $[7$, p. 511]) and

$$
\begin{aligned}
& \frac{E\left[\xi ; \xi_{1} ; \xi_{1}^{*}, \mathrm{X}\right]}{E\left[\xi_{0} ; \xi_{1} ; \xi_{1}^{*}, \mathrm{X}\right]} \\
& =\frac{\theta\left[J\left(\xi_{1} ; \mathrm{X}\right)_{1}-J(\xi ; \mathrm{X})_{1}-w_{1}(\mathrm{X}), \cdots, \mathrm{X}\right]}{\theta\left[J\left(\xi_{0} ; \mathrm{X}\right)_{1}-J(\xi ; \mathrm{X})_{1}-w_{1}(\mathrm{X}), \cdots, \mathrm{X}\right]} \\
& \quad \times \frac{\theta\left[J\left(\xi^{*} ; \mathrm{X}\right)_{1}-J\left(\xi_{0}, \mathrm{X}\right)_{1}-w_{1}(\mathrm{X}), \cdots, \mathrm{X}\right]}{\theta\left[J\left(\xi_{1}^{*} ; \mathrm{X}\right)_{1}-J(\xi, \mathrm{X})_{1}-w_{1}(\mathrm{X})_{1}, \cdots, \mathrm{X}\right]} \\
& \quad \times \exp \left\{\sum_{\alpha=1}^{\rho}\left[J\left(\xi_{1} ; \mathrm{X}\right)_{\alpha}-J\left(\xi_{1}^{*} ; \mathrm{X}\right)_{\alpha}\right]\left[J^{*}(\xi ; \mathrm{X})_{\alpha}-J^{*}\left(\xi^{*} ; \mathrm{X}\right)_{\alpha}\right]\right.
\end{aligned}
$$

where

$$
E\left[\xi ; \xi_{1}, \xi_{1}^{*}, \mathrm{X}\right]=\exp \left[\int_{\xi_{1}^{*}}^{\xi_{1}} H\left(\xi, \xi^{\prime}, \mathrm{X}\right) d \xi^{\prime}\right]
$$

\section{BIBLIOGRAPHY}

1. Stefan Bergman, Zur Theorie der ein- und mehrwertigen harmonischen Funktionen des dreidimensionalen Raumes, Math. Zeit. vol. 24 (1926) pp. 641-669.

2. - Zur Theorie der algebraischen Potentialfunktionen des dreidimensionalen Raumes, Math. Ann. vol. 99 (1928) pp. 629-659 and vol. 101 (1929) pp. 534-558.

3. - Zur Theorie der Funktionen die eine lineare partielle Differentialgleichung befriedigen, Rec. Math. (Mat. Sbornik) N.S. vol. 2 (1937) pp. 1169-1198. 
4. - Certain classes of analytic functions of two real variables, Trans. Amer. Math. Soc. vol. 57 (1945) pp. 299-331.

5. L. Fuchs, Die Periodicitatsmoduln der hyperelliptischen Integrale als Funktionen eines Parameters aufgefasst, Collected works, vol. 1, Berlin, 1904, pp. 241-281.

6. A. Sommerfeld, Über verzweigte Potentiale im Raume, Proc. London Math. Soc. vol. 28 (1897) pp. 395-429.

7. Karl Weierstrass, Vorlesungen über die Theorie der Abelschen Transcendenten, Collected Works, vol. 5, Berlin, 1902.

8. Morris Marden, Axisymmetric harmonic vectors, Amer. J. Math. vol. 67 (1945) pp. 109-122.

Brown UNIVERSITY, Providence, R. I. 